\title{
Chow motives without projectivity, II
}

\author{
by \\ Jörg Wildeshaus \\ Université Paris 13 \\ Sorbonne Paris Cité \\ LAGA, CNRS (UMR 7539) \\ F-93430 Villetaneuse \\ France \\ wildesh@math.univ-paris13.fr
}

September 27, 2018

\begin{abstract}
The purpose of this article is to provide a simplified construction of the intermediate extension of a Chow motive, provided a condition on absence of weights in the boundary is satisfied. We give a criterion, which guarantees the validity of the condition, and compare our new construction to the theory of the interior motive established earlier. We finish the article with a review of the known applications to the boundary of Shimura varieties.
\end{abstract}

Keywords: weight structures, boundary motive, interior motive, motivic intermediate extension, intersection motive.

Math. Subj. Class. (2010) numbers: 14F42 (14C25, 14F20, 14F32, 18E30, 19E15). 


\section{Contents}

1 Introduction

2 Rigidification of the intermediate extension 7

3 Motivic intermediate extension and interior motive 13

4 A criterion on absence of weights in the boundary 23

5 Examples: the boundary of certain Shimura varieties 29

\section{Introduction}

The aim of this article is to extend the main results from W3 to the context of motives over a base scheme $X$, taking into account and relying on the substantial progress the motivic theory has undergone since the writing of [loc. cit.].

As far as our aim is concerned, this progress concerns two main points: (1) the construction of the triangulated category $D M_{\mathrm{L}, c}(X)$ of motives over $X$ (generalizing the $\mathbb{Q}$-linear version of Voevodsky's definition for $X$ equal to a point, i.e., to the spectrum of a field), together with the formalism of six operations, (2) the construction of a weight structure on $D M_{\mathrm{b}, c}(X)$, compatible with the six operations.

As in [W3, the focus of our study is the absence of weights, and the guiding principle remains that absence of weights in motives associated to a boundary allows for the construction of a privileged extension of a given (Chow) motive. Even over a point, our approach via relative motives yields a new criterion (Theorem 4.8) on absence of weights in the boundary.

In the geometrical context of Siegel threefolds, it is that new criterion that is needed to control the weights W11. Indeed, the observation that earlier results concerning motives over a point were not sufficient to analyze the boundary of Siegel threefolds, can be seen as the main motivation of the present paper.

For a scheme $U$, which is separated and of finite type over a field $k$ (assumed to be of characteristic zero, to fix ideas), the boundary motive $\partial M_{g m}(U)$ of $U$ [W2] fits into an exact triangle

$(*) \quad \partial M_{g m}(U) \longrightarrow M_{g m}(U) \stackrel{u}{\longrightarrow} M_{g m}^{c}(U) \longrightarrow \partial M_{g m}(U)[1]$. 
Here, $M_{g m}(U)$ and $M_{g m}^{c}(U)$ denote the motive and the motive with compact support, respectively, of $U$, as defined by Voevodsky $\mathrm{Vo}$. Assuming in addition that $U$ is smooth over $k$, the objects $M_{g m}(U)$ and $M_{g m}^{c}(U)$ are of weights $\leq 0$ and $\geq 0$, respectively. The axioms imposed on a weight structure then formally imply that the morphism $u$ factors over a motive, which is pure of weight zero, in other words, $u$ factors over a Chow motive over $k$. However, such a factorization is by no means unique (for example, the motive of any smooth compactification of $U$ provides such a factorization). In this context, which is the one studied in W3, the "boundary" is the boundary motive $\partial M_{g m}(U)$, and any factorization of $u$ through a Chow motive is an "extension".

The above-mentioned progress, and more particularly, point (1) allows for what one might call "geometrical realizations" of the exact triangle $(*)$. Indeed, any open immersion $j: U \hookrightarrow X$ gives rise to an exact triangle

$$
(* *) \quad i_{*} i^{*} j_{*} \mathbb{1}_{U}[-1] \longrightarrow j_{!} \mathbb{1}_{U} \stackrel{m}{\longrightarrow} j_{*} \mathbb{1}_{U} \longrightarrow i_{*} i^{*} j_{*} \mathbb{1}_{U}
$$

of motives over $X$. Here, we denote by $i$ the closed immersion of the complement $Z$ of $U$ into $X$, and by $\mathbb{1}_{U}$ the structural motive over $U$. Provided that the structure morphism $a$ of $X$ is proper, the direct image $a_{*}$ of $(* *)$, i.e., the exact triangle

$$
a_{*} i_{*} i^{*} j_{*} \mathbb{1}_{U}[-1] \longrightarrow a_{*} j_{!} \mathbb{1}_{U} \stackrel{a_{*} m}{\longrightarrow} a_{*} j_{*} \mathbb{1}_{U} \longrightarrow a_{*} i_{*} i^{*} j_{*} \mathbb{1}_{U},
$$

is isomorphic to the dual of $(*)$.

Thanks to point (2), relative motives are endowed with weights. Independently of properness of the morphism $a$, the motives $j_{!} \mathbb{1}_{U}$ and $j_{*} \mathbb{1}_{U}$ over $X$ are of weights $\leq 0$ and $\geq 0$, respectively. Again, the axioms for weight structures imply that $m$ factors over a motive of weight zero. In this relative context, the "boundary" is the motive $i_{*} i^{*} j_{*} \mathbb{1}_{U}$ over $X$ (or equivalently, the motive $i^{*} j_{*} \mathbb{1}_{U}$ over $Z$ ), and any factorization of $m$ through a Chow motive is an "extension" of $\mathbb{1}_{U}$ (note that unless $X$ is smooth, too, the structural motive $\mathbb{1}_{X}$ is in general not pure of weight zero). If $a$ is proper, then the functor $a_{*}$ is weight exact. Applying it to a factorization of $m$ through a Chow motive over $X$, one therefore obtains a factorization of $u$ through a Chow motive over $k$.

More generally, any Chow motive $M_{U}$ over $U$ yields an exact triangle

$$
i_{*} i^{*} j_{*} M_{U}[-1] \longrightarrow j_{!} M_{U} \stackrel{m}{\longrightarrow} j_{*} M_{U} \longrightarrow i_{*} i^{*} j_{*} M_{U},
$$

$j_{!} M_{U}$ and $j_{*} M_{U}$ are of weights $\leq 0$ and $\geq 0$, respectively, and therefore, the morphism $m$ factors over a Chow motive over $X$. It is this context of relative motives which seems to be best adapted to our study. The above mentioned guiding principle relates absence of weights 0 and 1 in the boundary $i^{*} j_{*} M_{U}$ to the existence of a privileged extension of $M_{U}$ to a Chow motive over $X$, 
which is minimal in a precise sense among all such extensions. Furthermore, we are able to describe the sub-category of Chow motives over $X$ arising as such extensions. Our first main result is Theorem 2.2; it states that restriction $j^{*}$ from $X$ to $U$ induces an equivalence of categories

$$
j^{*}: C H M(X)_{i^{*} w \leq-1, i^{!} w \geq 1} \stackrel{\sim}{\longrightarrow} H M(U)_{\partial w \neq 0,1},
$$

where the left hand side denotes the full sub-category of Chow motives $M$ over $X$ such that $i^{*} M$ is of weights at most -1 , and $i^{!} M$ is of weights at least 1 , and the right hand side denotes the full sub-category of Chow motives $M_{U}$ over $U$ such that $i^{*} j_{*} M_{U}$ is without weights 0 and 1 .

It turns out that Theorem 2.2 is best proved in the abstract setting of triangulated categories $\mathcal{C}(U), \mathcal{C}(X)$ and $\mathcal{C}(Z)$ related by gluing, and equipped with weight structures compatible with the gluing. This is the setting of Section 2. The proof of Theorem 2.2 relies on Construction 2.3, which relates factorizations of $m: j_{!} M_{U} \rightarrow j_{*} M_{U}$, for objects $M_{U}$ of the heart $\mathcal{C}(U)_{w=0}$ of the weight structure on $\mathcal{C}(U)$, to weight filtrations of $i^{*} j_{*} M_{U}$. Theorem 2.2 establishes an equivalence

$$
j^{*}: \mathcal{C}(X)_{w=0, i^{*} w \leq-1, i^{\prime} w \geq 1} \stackrel{\sim}{\longrightarrow}(U)_{w=0, \partial w \neq 0,1},
$$

where source and target are defined in obvious analogy with the motivic situation. We can thus define the restriction of the intermediate extension to the category $\mathcal{C}(U)_{w=0, \partial w \neq 0,1}$

$$
j_{! *}: \mathcal{C}(U)_{w=0, \partial w \neq 0,1} \longleftrightarrow \mathcal{C}(X)_{w=0}
$$

as the composition of the inverse of the equivalence of Theorem 2.2, followed by the inclusion $\mathcal{C}(X)_{w=0, i^{*} w \leq-1, i^{\prime} w \geq 1} \longleftrightarrow \mathcal{C}(X)_{w=0}$ (Definition 2.4).

Theorem 2.2 allows us to provide important complements for the existing theory. First (Remark 2.6), the functor $j_{\text {!* }}$ is compatible with the theory developed in [W9, Sect. 2] when the additional hypothesis enabling the set-up of the latter, namely semi-primality of the category $\mathcal{C}(Z)_{w=0}$, is satisfied. Note that the functoriality properties of the theory from [loc. cit.] are intrinsically incomplete as the target of the intermediate extension is only a quotient of $\mathcal{C}(X)_{w=0}$. Definition 2.4 can thus be seen as providing a rigidification of the intermediate extension on the sub-category $\mathcal{C}(U)_{w=0, \partial w \neq 0,1}$ of $\mathcal{C}(U)_{w=0}$. This observation has rather useful consequences. When $\mathcal{C}(Z)_{w=0}$ is semi-primary, then by our very Definition 2.4, it is possible to read off $i^{*} j_{! *} M_{U}$ and $i^{!} j_{\text {!* }} M_{U}$ whether or not $M_{U}$ belongs to $\mathcal{C}(U)_{w=0, \partial w \neq 0,1}$ : indeed, $M_{U} \in \mathcal{C}(U)_{w=0, \partial w \neq 0,1}$ if and only if $i^{*} j_{!_{*}} M_{U}$ is of weights at most -1 , and $i^{!} j_{\text {!* }} M_{U}$ of weights at least 1 . In particular, the non-rigidified intermediate extension $j_{! *} M_{U}$ from [W9, Sect. 2] is rigid a posteriori, if $j_{! *} M_{U}$ belongs to the full sub-category $\mathcal{C}(X)_{w=0, i^{*} w \leq-1, i^{\prime} w>1}$ of $\mathcal{C}(X)_{w=0}$. Furthermore (Theorem [2.7), for $M_{U} \in \mathcal{C}(U)_{w=0, \partial w \neq 0,1}$, the interval $[\alpha, \beta] \supset[0,1]$ of weights avoided by $i^{*} j_{*} M_{U}$ can be determined directly from $i^{*} j_{!_{*}} M_{U}$ and $i^{!} j_{!_{*}} M_{U}$. 
For example, in the context studied in W11, i.e., of motives over Siegel threefolds, the condition on semi-primality is satisfied, and therefore, Theorem 2.7 applies.

Second, in the motivic context, Theorem 2.2 provides a criterion on absence of weights in the boundary, provided that the structure morphism $a$ of $X$ is proper. More generally, if $a$ is any proper morphism with source $X$, and $M_{U} \in C H M(U)_{\partial w \neq 0,1}$, then thanks to weight exactness of $a_{*}$, the motive $a_{*} i_{*} i^{*} j_{*} M_{U}$ is still without weights 0 and 1 . This means that condition W3, Asp. 2.3] is satisfied for the morphism

$$
a_{*} m: a_{*} j_{!} M_{U} \longrightarrow a_{*} j_{*} M_{U} .
$$

The principal aim of Section 3 is to spell out the consequences for our situation of the general theory developed in [W3, Sect. 2], given the validity of [W3, Asp. 2.3], and to relate them to the restriction of the intermediate extension to $C H M(U)_{\partial w \neq 0,1}$ (Theorems 3.4 3.6). Let us mention Theorem 3.5 in particular: any endomorphism of $(a \circ j) ! M_{U}$ or of $(a \circ j)_{*} M_{U}$ induces an endomorphism of the Chow motive $a_{*} j_{\text {!* }} M_{U}$. Theorem 3.5 applies in particular to endomorphisms "of Hecke type"; again, this general observation is used in particular in the geometrical context of Siegel threefolds [W11]. In case the proper morphism $a$ equals the structure morphism of $X$, the Chow motive $a_{*} j_{\text {!* }} M_{U}$ is defined to be the intersection motive of $U$ relative to $X$ with coefficients in $M_{U}$ (Definition 3.7). Given the state of the literature, it appeared useful to spell out the isomorphism between the dual of the interior motive [W3, Sect. 4] and the intersection motive. The comparison results from Proposition 3.8 onwards contain the earlier mentioned isomorphism between the dual of the exact triangle

$$
\partial M_{g m}(U) \longrightarrow M_{g m}(U) \stackrel{u}{\longrightarrow} M_{g m}^{c}(U) \longrightarrow \partial M_{g m}(U)[1]
$$

and the exact triangle

$$
a_{*} i_{*} i^{*} j_{*} \mathbb{1}_{U}[-1] \longrightarrow a_{*} j_{!} \mathbb{1}_{U} \stackrel{a_{*} m}{\longrightarrow} a_{*} j_{*} \mathbb{1}_{U} \longrightarrow a_{*} i_{*} i^{*} j_{*} \mathbb{1}_{U} .
$$

At this point, it is probably useful to recall that the quest for a motivic analogue of intersection cohomology started some thirty years ago, with the successful construction by Scholl of what should nowadays be seen as the intersection motive of modular curves [S]. This example, as other examples concerning Shimura varieties, will be discussed in Section 5. To the best of the author's knowledge, the only case where an intersection motive of "nonShimura type" was constructed over the field of definition of the geometric object, concerns arbitrary surfaces (with constant coefficients) [CM]; it may be worthwhile to note that this result appeared almost fifteen years after Scholl's!

A concrete difficulty arises when the defining condition of $C H M(U)_{\partial w \neq 0,1}$ needs to be checked for a concrete object of $C H M(U)$ : given a Chow motive 
$M_{U}$ over $U$, how to determine whether or not the motive $i^{*} j_{*} M_{U}$ is without weights 0 and 1? Section 4 gives what we think of as the optimal answer that can be given to date. Combining key results from [W10] and [W9, we prove Theorem 4.4, which we consider as our second main result: assume that the (generic) $\ell$-adic realization $R_{\ell, U}\left(M_{U}\right)$ of $M_{U}$ is concentrated in a single perverse degree, and that the motive $i^{*} j_{*} M_{U}$ is of Abelian type [W9]. Then whether or not $M_{U}$ belongs to $C H M(U)_{\partial w \neq 0,1}$ can be read off the perverse cohomology sheaves of $i^{*} j_{! *} R_{\ell, U}\left(M_{U}\right)$ and of $i^{!} j_{! *} R_{\ell, U}\left(M_{U}\right)$. If $M_{U} \in$ $C H M(U)_{\partial w \neq 0,1}$, then the precise interval $[\alpha, \beta] \supset[0,1]$ of weights avoided by $i^{*} j_{*} M_{U}$ can be determined from $i^{*} j_{! *} R_{\ell, U}\left(M_{U}\right)$ and $i^{!} j_{! *} R_{\ell, U}\left(M_{U}\right)$.

Chow motives have a tendancy to be auto-dual up to a shift and a twist; this is in any case true for the Chow motives occurring in the applications we have in mind, e.g., in the earlier mentioned analysis of the weights in the boundary of Siegel threefolds W11. Given that the criterion from Theorem 4.4 is compatible with duality, one may hope that the verification of a certain half of that criterion, for example the half concerning $i^{*} j_{! *} R_{\ell, U}\left(M_{U}\right)$, is sufficient, when $M_{U}$ is auto-dual. This hope is made explicit in Corollary 4.6. We think of this result as potentially very useful for other applications. For the sake of completeness, we combine Corollary 4.6 with the comparison from Section 3, the result is the earlier mentioned Theorem 4.8 .

The final Section 5 contains a complete review of the known applications of our theory to Shimura varieties. Let us point out that some of these cases are equally covered by a recent result of Vaish's [Va]. His approach replaces weight structures by weight truncations à la $\mathrm{S}$. Morel (but still relies on the main result from [W10]), thereby providing an alternative approach to the problem of rigidification of the intermediate extension. It is interesting to note that Vaish's result applies in certain situations where our condition on absence of weights 0 and 1 is not satisfied.

Part of this work was done while I was enjoying a délégation auprès $d u C N R S$, to which I wish to express my gratitude. I also wish to thank F. Déglise for useful discussions, and the referees for their comments, which contribued considerably to improve this article.

Conventions: Throughout the article, $F$ denotes a finite direct product of fields of characteristic zero, in other words, a commutative semi-simple Noetherian $\mathbb{Q}$-algebra. We fix a base scheme $\mathbb{B}$, which is of finite type over some excellent scheme of dimension at most two. By definition, schemes are $\mathbb{B}$-schemes which are separated and of finite type (in particular, they are excellent, and Noetherian of finite dimension), morphisms between schemes are separated morphisms of $\mathbb{B}$-schemes, and a scheme is nilregular if the underlying reduced scheme is regular. 
We use the triangulated, $\mathbb{Q}$-linear categories $D M_{\mathrm{E}, c}(X)$ of constructible Beilinson motives over $X$ CD2, Def. 15.1.1], indexed by schemes $X$ (always in the sense of the above conventions). In order to have an $F$-linear theory at one's disposal, one re-does the construction, but using $F$ instead of $\mathbb{Q}$ as coefficients [CD2, Sect. 15.2.5]. This yields triangulated, $F$-linear categories $D M_{\mathrm{L}, c}(X)_{F}$ satisfying the $F$-linear analogues of the properties of $D M_{\mathrm{E}, c}(X)$. In particular, these categories are pseudo-Abelian (see [H, Sect. 2.10]). Furthermore, the canonical functor of $F$-linear categories $D M_{\mathrm{D}, c}(X) \otimes_{\mathbb{Q}} F \rightarrow$ $D M_{\mathrm{E}, c}(X)_{F}$ is fully faithful [CD2, Sect. 14.2.20]. As in CD2, the symbol $\mathbb{1}_{X}$ is used to denote the unit for the tensor product in $D M_{\mathrm{L}, c}(X)_{F}$. We shall employ the full formalism of six operations developed in [loc. cit.]. The reader may choose to consult [H, Sect. 2] or [W5, Sect. 1] for concise presentations of this formalism.

Beilinson motives can be endowed with a canonical weight structure, thanks to the main results from $[\mathrm{H}]$ (see [B1, Prop. 6.5.3] for the case $X=\operatorname{Spec} k$, for a field $k$ of characteristic zero). We refer to it as the motivic weight structure. Following [W5, Def. 1.5], the category $C H M(X)_{F}$ of Chow motives over $X$ is defined as the heart $D M_{\mathrm{L}, c}(X)_{F, w=0}$ of the motivic weight structure on $D M_{\mathrm{E}, c}(X)_{F}$. It equals the pseudo-Abelian completion of $C H M(X)_{\mathbb{Q}} \otimes_{\mathbb{Q}} F$. According to $[\mathrm{H}$, Thm. 3.3 (ii)], the motivic weight structure on $D M_{\mathrm{L}, c}(X)_{F}$ is uniquely determined by the requirement that $f_{*} \mathbb{1}_{Y}(n)[2 n] \in C H M(X)_{F}$ whenever $n \in \mathbb{Z}$, and $f: Y \rightarrow X$ is a proper morphism with regular source $Y$.

When we assume a field $k$ to admit resolution of singularities, then it will be in the sense of [FV, Def. 3.4]: (i) for any separated $k$-scheme $X$ of finite type, there exists an abstract blow-up $Y \rightarrow X$ [FV, Def. 3.1] whose source $Y$ is smooth over $k$, (ii) for any pair of smooth, seperated $k$-schemes $X, Y$ of finite type, and any abstract blow-up $q: Y \rightarrow X$, there exists a sequence of blow-ups $p: X_{n} \rightarrow \ldots \rightarrow X_{1}=X$ with smooth centers, such that $p$ factors through $q$. We say that $k$ admits strict resolution of singularities, if in (i), for any given dense open subset $U$ of the smooth locus of $X$, the blow-up $q: Y \rightarrow X$ can be chosen to be an isomorphism above $U$, and such that arbitrary intersections of the irreducible components of the complement $Z$ of $U$ in $Y$ are smooth (e.g., $Z \subset Y$ a normal crossing divisor with smooth irreducible components).

\section{Rigidification of the intermediate extension}

Throughout this section, let us fix three $F$-linear pseudo-Abelian triangulated categories $\mathcal{C}(U), \mathcal{C}(X)$ and $\mathcal{C}(Z)$, the second of which is obtained from the others by gluing. This means that the three categories are equipped with six 
exact functors

$$
\begin{aligned}
& \mathcal{C}(U) \stackrel{j_{!}}{\longrightarrow} \mathcal{C}(X) \stackrel{i^{*}}{\longrightarrow} \mathcal{C}(Z) \\
& \mathcal{C}(U) \stackrel{j^{*}}{\longleftarrow} \mathcal{C}(X) \stackrel{i_{*}}{\longleftarrow} \mathcal{C}(Z) \\
& \mathcal{C}(U) \stackrel{j_{*}}{\longrightarrow} \mathcal{C}(X) \stackrel{i^{!}}{\longrightarrow} \mathcal{C}(Z)
\end{aligned}
$$

satisfying the axioms from [BBD, Sect. 1.4.3]. We assume that $\mathcal{C}(U), \mathcal{C}(X)$ and $\mathcal{C}(Z)$ are equipped with weight structures $w$ (the same letter for the three weight structures), and that the one on $\mathcal{C}(X)$ is actually obtained from the two others in a way compatible with the gluing, meaning that the left adjoints $j_{!}, j^{*}, i^{*}$ and $i_{*}$ respect the categories $\mathcal{C}(\bullet)_{w \leq 0}$, and the right adjoints $j^{*}, j_{*}, i_{*}$ and $i^{!}$respect the categories $\mathcal{C}(\bullet)_{w \geq 0}$. In particular, we have a fully faithful functor

$$
i_{*}: \mathcal{C}(Z)_{w=0} \longleftrightarrow \mathcal{C}(X)_{w=0}
$$

and a functor

$$
j^{*}: \mathcal{C}(X)_{w=0} \longrightarrow \mathcal{C}(U)_{w=0} .
$$

According to [W9, Prop. 2.5], the latter is full and essentially surjective. We shall need to understand its restriction to a certain sub-category of $\mathcal{C}(X)_{w=0}$.

Recall [W3, Def. 1.10] that an object $M$ is said to be without weights $m, \ldots, n$, or to avoid weights $m, \ldots, n$, for integers $m \leq n$, if it admits a weight filtration avoiding weights $m, \ldots, n$, i.e. [W3, Def. 1.6], if there is an exact triangle

$$
M_{\leq m-1} \longrightarrow M \longrightarrow M_{\geq n+1} \longrightarrow M_{\leq m-1}[1]
$$

with $M_{\leq m-1}$ of weights at most $m-1$, and $M_{\geq n+1}$ of weights at least $n+1$.

Definition 2.1. (a) Denote by $\mathcal{C}(U)_{w=0, \partial w \neq 0,1}$ the full sub-category of $\mathcal{C}(U)_{w=0}$ of objects $M_{U}$ such that $i^{*} j_{*} M_{U}$ is without weights 0 and 1 .

(b) Denote by $\mathcal{C}(X)_{w=0, i^{*} w \leq-1, i^{\prime} w \geq 1}$ the full sub-category of $\mathcal{C}(X)_{w=0}$ of objects $M$ such that $i^{*} M \in \mathcal{C}(Z)_{w \leq-1}$ and $i^{!} M \in \mathcal{C}(Z)_{w \geq 1}$.

Theorem 2.2. The restriction of $j^{*}$ to $\mathcal{C}(X)_{w=0, i^{*} w \leq-1, i^{\prime} w \geq 1}$ induces an equivalence of categories

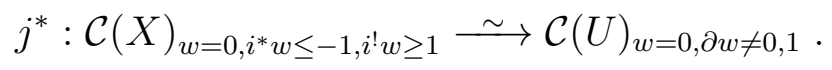

The proof of Theorem 2.2 relies on the following.

Construction 2.3. Let $M_{U} \in \mathcal{C}(U)_{w=0}$, and consider the morphism

$$
m: j_{!} M_{U} \longrightarrow j_{*} M_{U}
$$


in $\mathcal{C}(X)$. There is a canonical choice of cone of $m$, namely, the object $i_{*} i^{*} j_{*} M_{U}$. Any weight filtration of $i^{*} j_{*} M_{U}$

$$
C_{\leq 0} \stackrel{c_{-}}{\longrightarrow} i^{*} j_{*} M_{U} \stackrel{c_{+}}{\longrightarrow} C_{\geq 1} \stackrel{\delta}{\longrightarrow} C_{\leq 0}[1]
$$

(with $C_{\leq 0} \in \mathcal{C}(Z)_{w \leq 0}$ and $C_{\geq 1} \in \mathcal{C}(Z)_{w \geq 1}$ ) yields a diagram, whose 4-term lines and columns are exact triangles, and which we shall denote by the symbol (1)

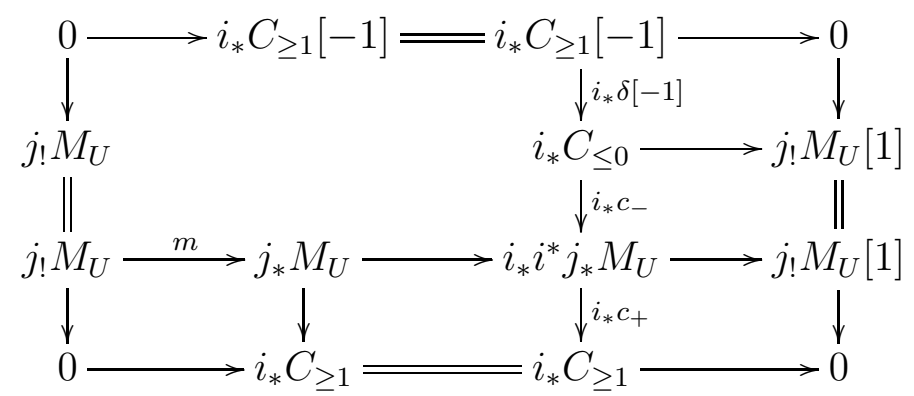

According to axiom TR4' of triangulated categories [BBD, Sect. 1.1.6], diagram (1) can be completed to give a diagram, denoted by (2)

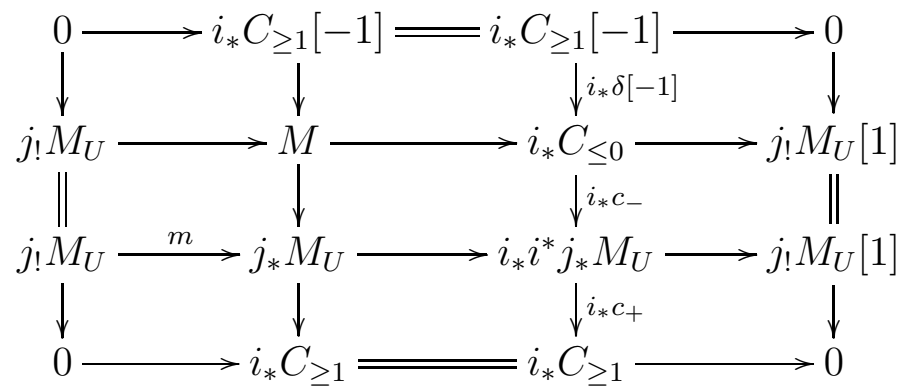

with $M \in \mathcal{C}(X)$. By the second row, and the second column of diagram (2), the object $M$ is simultaneously an extension of objects of weights $\leq 0$, and an extension of objects of weights $\geq 0$. It follows easily (c.f. [B1, Prop. 1.3 .3 3]) that $M$ belongs to both $\mathcal{C}(X)_{w \leq 0}$ and $\mathcal{C}(X)_{w \geq 0}$, and hence to $\mathcal{C}(X)_{w=0}$.

Applying the functors $j^{*}, i^{*}$, and $i^{!}$to $(2)$, we see that $j^{*} M=M_{U}$,

$$
i^{*} M \stackrel{\sim}{\longrightarrow} C_{\leq 0}, \quad \text { and } C_{\geq 1}[-1] \stackrel{\sim}{\longrightarrow} i^{!} M .
$$

Proof of Theorem 2.2. For $M_{U} \in \mathcal{C}(U)_{w=0}$ and a weight filtration

$$
C_{\leq 0} \longrightarrow i^{*} j_{*} M_{U} \longrightarrow C_{\geq 1} \longrightarrow C_{\leq 0}[1]
$$

of $i^{*} j_{*} M_{U}$, apply Construction 2.3 to get an extension $M \in \mathcal{C}(X)_{w=0}$ of $M_{U}$ to $X$. From the isomorphisms

$$
i^{*} M \stackrel{\sim}{\longrightarrow} C_{\leq 0} \text { and } C_{\geq 1}[-1] \stackrel{\sim}{\longrightarrow} i^{!} M,
$$

we see that $M \in \mathcal{C}(X)_{w=0, i^{*} w \leq-1, i^{!} w \geq 1}$ if and only if

$$
C_{\leq 0} \in \mathcal{C}(Z)_{w \leq-1} \quad \text { and } \quad C_{\geq 1} \in \mathcal{C}(Z)_{w \geq 2} .
$$


In particular, we see that any object in $\mathcal{C}(U)_{w=0, \partial w \neq 0,1}$ admits a pre-image under $j^{*}$ in $\mathcal{C}(X)_{w=0, i^{*} w \leq-1, i^{\prime} w \geq 1}$.

Conversely, any object $M$ from $\mathcal{C}(X)_{w=0}$ fits into a diagram of type (2)

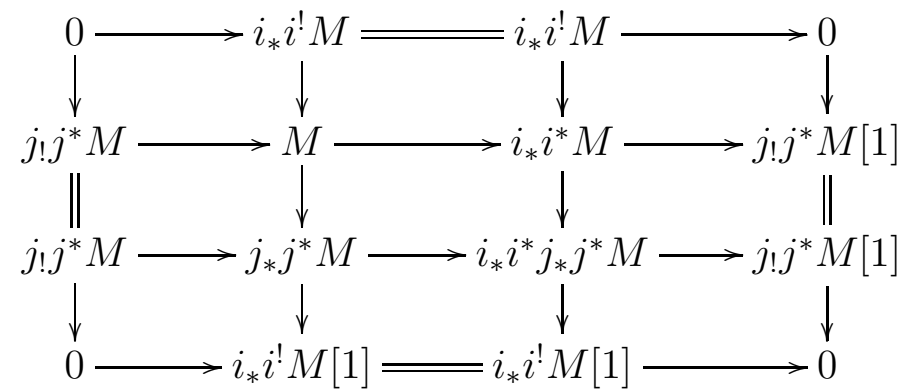

Its third column shows that $j^{*}$ maps $\mathcal{C}(X)_{w=0, i^{*} w \leq-1, i^{!} w>1}$ to $\mathcal{C}(U)_{w=0, \partial w \neq 0,1}$.

The restriction of $j^{*}$ therefore yields a well-defined functor

$$
j^{*}: \mathcal{C}(X)_{w=0, i^{*} w \leq-1, i^{!} w \geq 1} \longrightarrow \mathcal{C}(U)_{w=0, \partial w \neq 0,1},
$$

which is full [W9, Prop. 2.5] and essentially surjective. It remains to show that it is faithful, i.e., that a morphism $f$ in $\mathcal{C}(X)_{w=0, i^{*} w \leq-1, i^{i} w \geq 1}$ such that $j^{*} f=0$, equals zero.

Thus, let $M, M^{\prime} \in \mathcal{C}(X)_{w=0, i^{*} w \leq-1, i^{\prime} w \geq 1}, f: M \rightarrow M^{\prime}$, and assume that $j^{*} f=0$. This means that the composition of $f$ with the adjunction morphism $M^{\prime} \rightarrow j_{*} j^{*} M^{\prime}$ is zero. Given the exact localization triangle

$$
i_{*} i^{!} M^{\prime} \longrightarrow M^{\prime} \longrightarrow j_{*} j^{*} M^{\prime} \longrightarrow i_{*} i^{!} M^{\prime}[1],
$$

the morphism $f$ factors through $i_{*} i^{!} M^{\prime}$. Now $M$ is of weight zero, while $i^{!} M^{\prime}$, and hence $i_{*} i^{!} M^{\prime}$, is of strictly positive weights. By orthogonality, any morphism $M \rightarrow i_{*} i^{!} M^{\prime}$ is zero.

q.e.d.

Definition 2.4. Let the $F$-linear pseudo-Abelian triangulated categories $\mathcal{C}(U), \mathcal{C}(X)$ and $\mathcal{C}(Z)$ be related by gluing, and equipped with weight structures $w$ compatible with the gluing. Define the restriction of the intermediate extension to the category $\mathcal{C}(U)_{w=0, \partial w \neq 0,1}$

$$
j_{! *}: \mathcal{C}(U)_{w=0, \partial w \neq 0,1} \longleftrightarrow \mathcal{C}(X)_{w=0}
$$

as the composition of the inverse of the equivalence of Theorem 2.2, followed by the inclusion $\mathcal{C}(X)_{w=0, i^{*} w \leq-1, i^{!} w \geq 1} \longleftrightarrow \mathcal{C}(X)_{w=0}$.

Remark 2.5. Assume that contravariant auto-equivalences

$$
\mathbb{D} \text {. : } \mathcal{C}(\bullet) \stackrel{\sim}{\longrightarrow} \mathcal{C}(\bullet), \bullet \in\{U, X, Z\}
$$

are given, that they are compatible with the gluing $\left(\right.$ e.g., $\left.\mathbb{D}_{X} \circ j_{*} \cong j_{!} \circ \mathbb{D}_{U}\right)$ and with the weight structures $\left(\right.$ e.g., $\left.\mathbb{D}_{X} \mathcal{C}(X)_{w \geq 0}=\mathcal{C}(X)_{w<0}\right)$. From the isomorphisms $\mathbb{D}_{Z} \circ i^{*} \circ j_{*} \cong i^{!} \circ j_{!} \circ \mathbb{D}_{U}$ and $i^{!} \circ j_{!} \cong i^{*} \circ j_{*}[-1]$, it follows first that the functor $\mathbb{D}_{U}$ respects the category $\mathcal{C}(U)_{w=0, \partial w \neq 0,1}$. Similarly, the functor $\mathbb{D}_{X}$ respects the category $\mathcal{C}(X)_{w=0, i^{*} w \leq-1, i^{i} w \geq 1}$. 

that

It then follows formally from Definition $\left[2.4\right.$, and from $\mathbb{D}_{U} \circ j^{*} \cong j^{*} \circ \mathbb{D}_{X}$

$$
\mathbb{D}_{X} \circ j_{! *} \cong j_{! *} \circ \mathbb{D}_{U}
$$

In other words, the restriction of the intermediate extension to $\mathcal{C}(U)_{w=0, \partial w \neq 0,1}$ is compatible with local duality.

Remark 2.6. (a) Assume that some composition of morphisms

$$
i_{*} N \longrightarrow M \longrightarrow i_{*} N
$$

gives the identity on $i_{*} N$, for some object $N$ of $\mathcal{C}(Z)_{w=0}$. Then the adjunction properties of $i^{*}, i_{*}$ and $i^{!}$, and in particular, the identifications $i^{*} i_{*}=\operatorname{id} d_{\mathcal{C}(Z)}$ and $\operatorname{id}_{\mathcal{C}(Z)}=i^{!} i_{*}$, show that $N$ is a direct factor of both $i^{!} M$ and $i^{*} M$, and that the restriction of the composition $i^{!} M \rightarrow i^{*} M$ of the adjunction morphisms to this direct factor is the identity.

We obtain that objects in $\mathcal{C}(X)_{w=0, i^{*} w<-1, i^{i} w>1}$ do not admit non-zero direct factors belonging to the image of $\mathcal{C}(\bar{Z})_{w=0}$ under the functor $i_{*}$. This justifies Definition 2.4: the intermediate extension of $M_{U} \in \mathcal{C}(U)_{w=0, \partial w \neq 0,1}$ is indeed an extension of $M_{U}$ not admitting non-zero direct factors belonging to the image of $i_{*}$.

(b) More generally, for $M \in \mathcal{C}(X)_{w=0, i^{*} w \leq-1, i^{i} w>1}$ and $N \in \mathcal{C}(Z)_{w=0}$, any morphism $M \rightarrow i_{*} N$ is zero, and so is any morphism $i_{*} N \rightarrow M$.

(c) Following [W9, Def. 1.6 (a)], denote by $\mathfrak{g}$ the two-sided ideal of $\mathcal{C}(X)_{w=0}$ generated by

$$
\operatorname{Hom}_{\mathcal{C}(X)_{w=0}}\left(M, i_{*} N\right) \text { and } \operatorname{Hom}_{\mathcal{C}(X)_{w=0}}\left(i_{*} N, M\right),
$$

for all objects $(M, N)$ of $\mathcal{C}(X)_{w=0} \times \mathcal{C}(Z)_{w=0}$, such that $M$ admits no nonzero direct factor belonging to the image of $i_{*}$. Denote by $\mathcal{C}(X)_{w=0}^{u}$ the quotient of $\mathcal{C}(X)_{w=0}$ by $\mathfrak{g}$. From (b), we see that $\mathfrak{g}\left(M, M^{\prime}\right)=0$, for any $M, M^{\prime} \in \mathcal{C}(X)_{w=0, i^{*} w \leq-1, i^{\prime} w \geq 1}$. Thus, the quotient functor $\mathcal{C}(X)_{w=0} \longrightarrow$ $\mathcal{C}(X)_{w=0}^{u}$ induces an auto-equivalence on $\mathcal{C}(X)_{w=0, i^{*} w \leq-1, i^{\prime} w \geq 1}$.

(d) From the above, we see that Definition 2.4 is compatible with the theory developed in [W9, Sect. 2] when the hypotheses enabling the set-up of the latter are satisfied. More precisely, assume in addition that $\mathcal{C}(Z)_{w=0}$ is semiprimary [AK, Déf. 2.3.1]. Then

$$
j_{! *}: \mathcal{C}(U)_{w=0} \longleftrightarrow \mathcal{C}(X)_{w=0}^{u}
$$

is defined on the whole of $\mathcal{C}(U)_{w=0}$ [W9, Def. 2.10]. Parts (a) and (c) of the present Remark, and [W9, Summ. 2.12 (a) (1)] show that the diagram

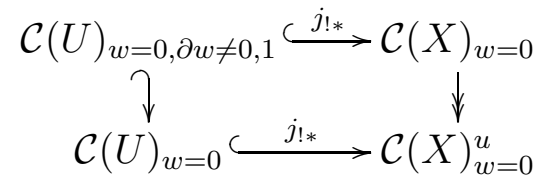


is commutative. Thus, $j_{\text {!* }}: \mathcal{C}(U)_{w=0, \partial w \neq 0,1} \hookrightarrow \mathcal{C}(X)_{w=0}$ from Definition 2.4 is indeed the restriction of $j_{!_{*}}: \mathcal{C}(U)_{w=0} \hookrightarrow \mathcal{C}(X)_{w=0}^{u}$ from [W9, Sect. 2], when the latter is defined, to $\mathcal{C}(U)_{w=0, \partial w \neq 0,1}$.

Theorem 2.7. Let $M_{U} \in \mathcal{C}(U)_{w=0}$.

(a) Assume that the category $\mathcal{C}(Z)_{w=0}$ is semi-primary, so that the functor $j_{\text {!* }}$ is defined on the whole of $\mathcal{C}(U)_{w=0}\left[W 9\right.$, Sect. 2]. Then the object $M_{U}$ belongs to $\mathcal{C}(U)_{w=0, \partial w \neq 0,1}$ if and only if $j_{!_{*}} M_{U}$ belongs to $\mathcal{C}(X)_{w=0, i^{*} w \leq-1, i^{!} w \geq 1}$.

(b) Assume that $M_{U}$ belongs to $\mathcal{C}(U)_{w=0, \partial w \neq 0,1}$. Let $\alpha \leq 0$ and $\beta \geq 1$ be two integers. Then $i^{*} j_{*} M_{U}$ is without weights $\alpha, \alpha+1, \ldots, \beta$ if and only if

$$
i^{*} j_{!_{*}} M_{U} \in \mathcal{C}(Z)_{w \leq \alpha-1} \quad \text { and } \quad i^{!} j_{! *} M_{U} \in \mathcal{C}(Z)_{w \geq \beta} .
$$

Proof. The "if" part from (a) is Theorem 2.2, and its "only if" part is Remark 2.6 (d).

As for (b), note that by definition, Construction 2.3 for $M_{U}$ and a weight filtration

$$
C_{\leq-1} \longrightarrow i^{*} j_{*} M_{U} \longrightarrow C_{\geq 2} \longrightarrow C_{\leq-1}[1]
$$

of $i^{*} j_{*} M_{U}$ avoiding weights 0 and 1 yields the extension $M=j_{\text {!* }} M_{U}$ of $M_{U}$ to $X$. The claim thus follows from the isomorphisms

$$
i^{*} j_{! *} M_{U} \stackrel{\sim}{\longrightarrow} C_{\leq-1} \text { and } C_{\geq 2}[-1] \stackrel{\sim}{\longrightarrow} i^{!} j_{! *} M_{U} .
$$

q.e.d.

Remark 2.8. (a) As the attentive reader will have remarked already, the formalism could have been developed on larger sub-categories of $\mathcal{C}(X)$ and $\mathcal{C}(U)$, at the cost of losing its inherent auto-duality. More precisely, define full sub-categories $\mathcal{C}(U)_{w=0, \partial w \neq 0}$ and $\mathcal{C}(U)_{w=0, \partial w \neq 1}$ of $\mathcal{C}(U)_{w=0}$, and $\mathcal{C}(X)_{w=0, i^{*} w \leq-1}$ and $\mathcal{C}(X)_{w=0, i^{\prime} w \geq 1}$ of $\mathcal{C}(X)_{w=0}$ in the obvious way. Then as in Theorem $[2.2$,

$$
j^{*}: \mathcal{C}(X)_{w=0, i^{*} w \leq-1} \stackrel{\sim}{\longrightarrow} \mathcal{C}(U)_{w=0, \partial w \neq 0}
$$

and

$$
j^{*}: \mathcal{C}(X)_{w=0, i^{\prime} w \geq 1} \stackrel{\sim}{\mathcal{C}}(U)_{w=0, \partial w \neq 1},
$$

allowing to define the restrictions $j_{!_{*}}$ of the intermediate extension to both $\mathcal{C}(U)_{w=0, \partial w \neq 0}$ and $\mathcal{C}(U)_{w=0, \partial w \neq 1}$. Local duality as in Remark 2.5 exchanges the two constructions. Parts (a), (c), and (d) (but not (b)) of Remark 2.6 apply with the most obvious modifications.

(b) Even if it will not be needed in the sequel of this article, it is worthwhile to spell out the modified version of Theorem 2.7. Let $M_{U} \in \mathcal{C}(U)_{w=0}$. Then

$$
M_{U} \in \mathcal{C}(U)_{w=0, \partial w \neq 0} \Longleftrightarrow j_{! *} M_{U} \in \mathcal{C}(X)_{w=0, i^{*} w \leq-1}
$$

and

$$
M_{U} \in \mathcal{C}(U)_{w=0, \partial w \neq 1} \Longleftrightarrow j_{! *} M_{U} \in \mathcal{C}(X)_{w=0, i^{\prime} w \geq 1}
$$


provided that $\mathcal{C}(Z)_{w=0}$ is semi-primary. More interestingly, part (b) of Theorem 2.7 can be separated into two statements: let $\alpha \leq 0$ and $\beta \geq 1$. Assume that $M_{U} \in \mathcal{C}(U)_{w=0, \partial w \neq 0}$ or $M_{U} \in \mathcal{C}(U)_{w=0, \partial w \neq 1}$. Then $i^{*} j_{*} M_{U}$ is without weights $\alpha, \alpha+1, \ldots, 0$ if and only if

$$
i^{*} j_{! *} M_{U} \in \mathcal{C}(Z)_{w \leq \alpha-1},
$$

and $i^{*} j_{*} M_{U}$ is without weights $1,2, \ldots, \beta$ if and only if

$$
i^{!} j_{! *} M_{U} \in \mathcal{C}(Z)_{w \geq \beta} \text {. }
$$

\section{Motivic intermediate extension and inte- rior motive}

The purpose of the present section is to connect Section 2 to the theory developed in [W3, Sect. 2]. The main results are Theorems [3.4, 3.5 and 3.6, these concern the motivic intermediate extension, and are formal analogues of the main results from [W3, Sect. 4] on the interior motive, defined and studied in [loc. cit.]. When the base scheme $\mathbb{B}$ is the spectrum of a field admitting strict resolution of singularities, then the analogy is not just formal: Corollary 3.10 establishes an isomorphism between the dual of the interior motive and the direct image of the motivic intermediate extension under the structure morphism, provided the latter is proper and that weights 0 and 1 are avoided.

Let $X$ be a scheme (in the sense of our Introduction), and $j: U \hookrightarrow X$ an open immersion with complement $i: Z \hookrightarrow X$. Thanks to localization CD2, Sect. 2.3], and to compatibility of the motivic weight structure with gluing [H, Thm. 3.8], Theorem 2.2 applies to the categories $\mathcal{C}(\bullet)=D M_{\mathrm{L}, c}(\bullet)_{F}$, for $\bullet=U, X, Z$. We write $C H M(U)_{F, \partial w \neq 0,1}$ for $D M_{\mathrm{E}, c}(U)_{F, w=0, \partial w \neq 0,1}$, and $C H M(X)_{F, i^{*} w<-1, i^{\prime} w \geq 1}$ for $D M_{\mathrm{E}, c}(X)_{F, w=0, i^{*} w \leq-1, i^{\prime} w \geq 1}$. In this motivic context, Remark 2.6 (d) says that the functor $j_{\text {!* }}$ from Definition 2.4 equals the restriction to $C H M(U)_{F, \partial w \neq 0,1}$ of the motivic intermediate extension, when the latter is defined; note that this is the case in the context of motives of Abelian type, studied in [W9, Sect. 5] (see our Section 4).

Proposition 3.1. Let $a: X \rightarrow \mathbb{A}$ a proper morphism of schemes, and $M_{U}$ a Chow motive over $U$ belonging to $C H M(U)_{F, \partial w \neq 0,1}$. Denote by $m$ the morphism $j_{!} M_{U} \rightarrow j_{*} M_{U}$.

(a) The image

$$
a_{*} j_{!} M_{U} \stackrel{a_{*} m}{\longrightarrow} a_{*} j_{*} M_{U} \longrightarrow a_{*} i_{*} i^{*} j_{*} M_{U} \longrightarrow a_{*} j_{!} M_{U}[1]
$$

under $a_{*}$ of the triangle

$$
j_{!} M_{U} \stackrel{m}{\longrightarrow} j_{*} M_{U} \longrightarrow i_{*} i^{*} j_{*} M_{U} \longrightarrow j_{!} M_{U}[1]
$$


in $D M_{\mathrm{E}, c}(\mathbb{A})_{F}$ is exact.

(b) The motive $a_{*} i_{*} i^{*} j_{*} M_{U} \in D M_{\mathrm{E}, c}(\mathbb{A})_{F}$ is without weights 0 and 1 .

Proof. (a): Indeed, the triangle

$$
j_{!} M_{U} \stackrel{m}{\longrightarrow} j_{*} M_{U} \longrightarrow i_{*} i^{*} j_{*} M_{U} \longrightarrow j_{!} M_{U}[1]
$$

is exact.

(b): The morphism $a \circ i$ being proper, we have $(a \circ i)_{!}=(a \circ i)_{*}=a_{*} i_{*}$. Thus, $a_{*} i_{*}$ is weight exact. It therefore transforms any weight filtration avoiding weights 0 and 1 into the same kind of weight filtration. q.e.d.

Thus, [W3, Asp. 2.3] is satisfied, with $\mathcal{C}=D M_{\mathrm{Б}, c}(\mathbb{A})_{F}, u=a_{*} m$ : $a_{*} j_{!} M_{U} \rightarrow a_{*} j_{*} M_{U}$, and $C=a_{*} i_{*} i^{*} j_{*} M_{U}[-1]$. Consequently, the theory developed in [W3, Sect. 2] applies.

Definition 3.2 (cmp. [W3, Def. 2.1]). Denote by $D M_{\mathrm{Б}, c}(\mathbb{A})_{F, w<0, \neq-1}$ the full sub-category of $D M_{\mathrm{E}, c}(\mathbb{A})_{F, w \leq 0}$ of objects without weight -1 , and by $D M_{\mathrm{E}, c}(\mathbb{A})_{F, w \geq 0, \neq 1}$ the full sub-category of $D M_{\mathrm{E}, c}(\mathbb{A})_{F, w \geq 0}$ of objects without weight 1 .

Proposition 3.3 ([W3, Prop. 2.2]). The inclusions

$$
\iota_{-}: C H M(\mathbb{A})_{F} \longleftrightarrow D M_{\mathrm{E}, c}(\mathbb{A})_{F, w \leq 0, \neq-1}
$$

and

$$
\iota_{+}: C H M(\mathbb{A})_{F} \hookrightarrow D M_{\mathrm{Б}, c}(\mathbb{A})_{F, w \geq 0, \neq 1}
$$

admit a left adjoint

$$
\mathrm{Gr}_{0}: D M_{\mathrm{E}, c}(\mathbb{A})_{F, w \leq 0, \neq-1} \longrightarrow C H M(\mathbb{A})_{F}
$$

and a right adjoint

$$
\mathrm{Gr}_{0}: D M_{\mathrm{E}, c}(\mathbb{A})_{F, w \geq 0, \neq 1} \longrightarrow C H M(\mathbb{A})_{F},
$$

respectively. Both adjoints map objects (and morphisms) to the term of weight zero of a weight filtration avoiding weight -1 and 1 , respectively. The compositions $\mathrm{Gr}_{0} \circ \iota_{-}$and $\mathrm{Gr}_{0} \circ \iota_{+}$both equal the identity on $C H M(\mathbb{A})_{F}$.

Theorem 3.4. Let $a: X \rightarrow \mathbb{A}$ a proper morphism.

(a) The essential image of the restriction of the functor $a_{*} j_{\text {! }}$ to the subcategory $C H M(U)_{F, \partial w \neq 0,1}$ is contained in $D M_{\mathrm{B}, c}(\mathbb{A})_{F, w \leq 0, \neq-1}$, inducing a functor

$$
(a \circ j)_{!}=a_{*} j_{!}: C H M(U)_{F, \partial w \neq 0,1} \longrightarrow D M_{\mathrm{B}, c}(\mathbb{A})_{F, w \leq 0, \neq-1} .
$$

More precisely, if $M_{U} \in C H M(U)_{F}$ is such that $i^{*} j_{*} M_{U}$ avoids weights $\alpha, \alpha+$ $1, \ldots, \beta$, for integers $\alpha \leq 0$ and $\beta \geq 1$, then

$$
a_{*} i_{*} i^{*} j_{! *} M_{U}[-1] \longrightarrow a_{*} j_{!} M_{U} \longrightarrow a_{*} j_{! *} M_{U} \longrightarrow a_{*} i_{*} i^{*} j_{! *} M_{U}
$$


is a weight filtration of $a_{*} j_{!} M_{U}$ avoiding weights $\alpha-1, \alpha, \ldots,-1$.

(b) The essential image of the restriction of the functor $a_{*} j_{*}$ to the subcategory $C H M(U)_{F, \partial w \neq 0,1}$ is contained in $D M_{\mathrm{E}, c}(\mathbb{A})_{F, w \geq 0, \neq 1}$, inducing a functor

$$
(a \circ j)_{*}=a_{*} j_{*}: C H M(U)_{F, \partial w \neq 0,1} \longrightarrow D M_{\mathrm{E}, c}(\mathbb{A})_{F, w \geq 0, \neq 1} .
$$

More precisely, if $M_{U} \in C H M(U)_{F}$ is such that $i^{*} j_{*} M_{U}$ avoids weights $\alpha, \alpha+$ $1, \ldots, \beta$, for integers $\alpha \leq 0$ and $\beta \geq 1$, then

$$
a_{*} j_{! *} M_{U} \longrightarrow a_{*} j_{*} M_{U} \longrightarrow a_{*} i_{*} i^{!} j_{! *} M_{U}[1] \longrightarrow a_{*} j_{! *} M_{U}[1]
$$

is a weight filtration of $a_{*} j_{*} M_{U}$ avoiding weights $1,2, \ldots, \beta$.

(c) There are canonical isomorphisms of functors

$\mathrm{Gr}_{0} \circ(a \circ j)_{!}=\mathrm{Gr}_{0} \circ a_{*} j_{!} \stackrel{\sim}{\longrightarrow} a_{*} j_{! *} \quad$ and $\quad a_{*} j_{! *} \stackrel{\sim}{\longrightarrow} \mathrm{Gr}_{0} \circ a_{*} j_{*}=\mathrm{Gr}_{0} \circ(a \circ j)_{*}$ on the category $C H M(U)_{F, \partial w \neq 0,1}$. Their composition equals the value of the functor $\mathrm{Gr}_{0} \circ a_{*}$ at the restriction of the natural transformation $m: j_{!} \rightarrow j_{*}$ to $C H M(U)_{F, \partial w \neq 0,1}$; in particular,

$$
\mathrm{Gr}_{0} \circ\left(a_{*} \circ m\right): \mathrm{Gr}_{0} \circ(a \circ j)_{!} \longrightarrow \mathrm{Gr}_{0} \circ(a \circ j)_{*}
$$

is an isomorphism of functors on $C H M(U)_{F, \partial w \neq 0,1}$.

Proof. Let $M_{U} \in C H M(U)_{F, \partial w \neq 0,1}$. By definition (and Theorem 2.2), the motive $j_{!_{*}} M_{U}$ belongs to $C H M(X)_{F, i^{*} w \leq-1, i^{i} w \geq 1}$. Thus, the exact triangles

$$
i_{*} i^{*} j_{! *} M_{U}[-1] \longrightarrow j_{!} M_{U} \longrightarrow j_{! *} M_{U} \longrightarrow i_{*} i^{*} j_{! *} M_{U}
$$

and

$$
j_{! *} M_{U} \longrightarrow j_{*} M_{U} \longrightarrow i_{*} i^{!} j_{! *} M_{U}[1] \longrightarrow j_{! *} M_{U}[1]
$$

are weight filtrations (of $j_{!} M_{U}$ ) avoiding weight -1 , and (of $j_{*} M_{U}$ ) avoiding weight 1, respectively. An analogous statement is therefore true for their images under the weight exact functor $a_{*}$ (recall that $a$ is assumed to be proper). Together with Proposition 3.3, this shows part (c) of the statement, as well as the first claims of parts (a) and (b). The second, more precise claims follow from Theorem 2.7 (b).

q.e.d.

At first sight, it may thus appear that the theory from [W3, Sect. 2] does not add much to what we get by explicit identification of the weight filtrations. But then, note the following.

Theorem 3.5. Let $a: X \rightarrow \mathbb{A}$ a proper morphism. Then for any Chow motive $M_{U} \in C H M(U)_{F, \partial w \neq 0,1}$, the Chow motive $a_{*} j_{! *} M_{U} \in C H M(\mathbb{A})_{F}$ behaves functorially with respect to both motives $(a \circ j) ! M_{U}$ and $(a \circ j)_{*} M_{U}$. In particular, any endomorphism of $(a \circ j) ! M_{U}$ or of $(a \circ j)_{*} M_{U}$ induces an endomorphism of $a_{*} j_{! *} M_{U}$. 
Proof. This follows from the functorial identities from Theorem 3.4 (c).

q.e.d.

Theorem 3.6. Let $M_{U} \in C H M(U)_{F, \partial w \neq 0,1}, a: X \rightarrow \mathbb{A}$ a proper morphism, and assume given a factorization $(a \circ j) ! M_{U} \rightarrow M_{\mathbb{A}} \rightarrow(a \circ j)_{*} M_{U}$ of the morphism $a_{*} m:(a \circ j) ! M_{U} \rightarrow(a \circ j)_{*} M_{U}$ through an object $M_{\mathbb{A}}$ of $C H M(\mathbb{A})_{F}$. Then $a_{*} j_{!_{*}} M_{U}$ is canonically identified with a direct factor of $M_{\mathbb{A}}$, admitting a canonical complement.

Proof. This is [W3, Cor. 2.5].

q.e.d.

The theory applies in particular when $a$ equals the structure morphism from $X$ to the base scheme $\mathbb{B}$.

Definition 3.7. Assume that $X$ is proper over $\mathbb{A}=\mathbb{B}$. Denote by $a$ : $X \rightarrow \mathbb{B}$ the structure morphism of $X$. Let $M_{U} \in C H M(U)_{F, \partial w \neq 0,1}$. We call $a_{*} j_{\text {!* }} M_{U}$ the intersection motive of $U$ relative to $X$ with coefficients in $M_{U}$.

Our terminology is motivated by one of the main results of [W9]. It states that on Chow motives of Abelian type, the cohomological Betti [Ay, Déf. 2.1] and $\ell$-adic realizations [CD3, Sect. 7.2, see in part. Rem. 7.2.25] are compatible with intermediate extensions (of motives, and of perverse sheaves). For details, we refer to [W9, Thm. 7.2]. Since the realizations are compatible with direct images, they therefore map $a_{*} j_{! *} M_{U}$ to intersection cohomology whenever $M_{U}$ is a Chow motive of Abelian type.

It turns out that the comparison results from CD2] allow to connect the above to the notion of interior motive.

Proposition 3.8. Assume that $\mathbb{A}=\mathbb{B}=$ Spec $k$ for a field $k$ admitting strict resolution of singularities. Assume also that the structure morphism $a: X \rightarrow \mathbb{B}$ is proper, and that its restriction $a \circ j$ to $U \subset X$ is smooth. Let $\pi: \mathrm{C} \rightarrow U$ proper and smooth (hence $\mathrm{C}$ is smooth over $k$ ). Assume $C$ to be quasi-projective over $k$. Consider the Chow motive $\pi_{*} \mathbb{1}_{\mathrm{C}}$ over $U$. Then the morphism

$$
a_{*} m:(a \circ j) ! \pi_{*} \mathbb{1}_{\mathrm{C}} \longrightarrow(a \circ j)_{*} \pi_{*} \mathbb{1}_{\mathrm{C}}
$$

is canonically and $\mathrm{CH}_{d_{\mathrm{C}}}\left(\mathrm{C} \times{ }_{U} \mathrm{C}\right)$-equivariantly isomorphic $\left(d_{\mathrm{C}}:=\right.$ the absolute dimension of $\mathrm{C}$ ) to the dual of the morphism

$$
u: M_{g m}(\mathrm{C}) \longrightarrow M_{g m}^{c}(\mathrm{C})
$$

in $D M_{g m}(k)_{F}$ [Vo, pp. 223-224].

A few words of explanation are in order. First, by [CD2, Cor. 16.1.6], the triangulated category $D M_{\mathrm{Б}, c}(\operatorname{Spec} k)_{F}$ is identified with the $F$-linear version of the triangulated category of geometrical motives $D M_{g m}(k)_{F} \overline{\mathrm{VO}}$, 
p. 192] (see [And, Sect. 17.1.3]). Second, the duality in question is the functor mapping $N$ to

$$
N^{*}:=\underline{\operatorname{Hom}}\left(N, \mathbb{1}_{\mathrm{Spec} k}\right) .
$$

Third, equivariance under the Chow group $\mathrm{CH}_{d_{\mathrm{C}}}\left(\mathrm{C} \times_{U} \mathrm{C}\right)$ refers to the following. According to [CD2, Cor. 14.2.14],

$$
\operatorname{End}_{C H M(U)_{F}}\left(\pi_{*} \mathbb{1}_{\mathrm{C}}\right)=\mathrm{CH}_{d_{\mathrm{C}}}\left(\mathrm{C} \times_{U} \mathrm{C}\right) \otimes_{\mathbb{Z}} F
$$

meaning that the Chow group $\mathrm{CH}_{d_{\mathrm{C}}}\left(\mathrm{C} \times_{U} \mathrm{C}\right)$ acts on $\pi_{*} \mathbb{1}_{\mathrm{C}} \in D M_{\mathrm{E}, c}(U)_{F}$. Hence the morphism $a_{*} m$ is $\mathrm{CH}_{d_{\mathrm{C}}}\left(\mathrm{C} \times_{U} \mathrm{C}\right)$-equivariant. As for the action on $M_{g m}(\mathrm{C})$, on $M_{g m}^{c}(\mathrm{C})$, and the equivariance of $u$, we refer to [D, Thm. 5.23] and [CD1, Ex. 4.12, Ex. 7.15]. tion

Remark 3.9. (a) According to [L, Prop. 5.19, Cor. 6.14], the identifica-

$$
\operatorname{End}_{C H M(U)_{F}}\left(\pi_{*} \mathbb{1}_{\mathrm{C}}\right)=\mathrm{CH}_{d_{\mathrm{C}}}\left(\mathrm{C} \times_{U} \mathrm{C}\right) \otimes_{\mathbb{Z}} F
$$

is compatible with composition. Thus, the action of the ring $\mathrm{CH}_{d_{\mathrm{C}}}\left(\mathrm{C} \times_{U} \mathrm{C}\right)$ on $\pi_{*} \mathbb{1}_{\mathrm{C}}$ is by correspondences in the classical sense.

(b) Note that since we assumed $C$ to be proper and smooth over $U$, the Chow motive $\pi_{*} \mathbb{1}_{\mathrm{C}}$ is smooth in the sense of [L]. According to [F, Thm. 3.17], if $U$ is quasi-projective over $k$, then the comparision statement

$$
\operatorname{End}_{C H M(U)_{F}}\left(\pi_{*} \mathbb{1}_{\mathrm{C}}\right)=\mathrm{CH}_{d_{\mathrm{C}}}\left(\mathrm{C} \times_{U} \mathrm{C}\right) \otimes_{\mathbb{Z}} F
$$

continues to hold if $C$ is assumed to be proper over $U$, and to remain quasiprojective and smooth over $k$.

Proof of Proposition 3.8. The morphism $a_{*} m$ coincides with the value of the natural transformation of functors

$$
(a \circ j \circ \pi)_{!} \longrightarrow(a \circ j \circ \pi)_{*}
$$

on $\mathbb{1}_{\mathrm{C}}$, since both $\pi$ and $a$ are proper [CD2, Thm. 2.2.14 (2)]. Fix a projective and smooth compactification $\overline{\mathrm{C}}$ of $\mathrm{C}$ over $k$, write $j^{\prime}$ for the open immersion of $\mathrm{C}$ into $\overline{\mathrm{C}}$, and $c$ for the structure morphism of $\overline{\mathrm{C}}$. Thus, $c \circ j^{\prime}=a \circ j \circ \pi$ is the structure morphism of $\mathrm{C}$. Then $a_{*} m$ equals $c_{*} m^{\prime}$, for the morphism $m^{\prime}: j_{!}^{\prime} \mathbb{1}_{\mathrm{C}} \rightarrow j_{*}^{\prime} \mathbb{1}_{\mathrm{C}}$ in $D M_{\mathrm{E}, c}(\overline{\mathrm{C}})_{F}$, and can be factorized as follows:

$$
a_{*} m=c_{*} a d j_{1} \circ c_{*} a d j_{2}: c_{*} j_{!}^{\prime} \mathbb{1}_{\mathrm{C}} \stackrel{c_{*} a d j_{2}}{\longrightarrow} c_{*} \mathbb{1}_{\overline{\mathrm{C}}} \stackrel{c_{*} a d j_{1}}{\longrightarrow} c_{*} j_{*}^{\prime} \mathbb{1}_{\mathrm{C}},
$$

where $a d j_{1}: \mathbb{1}_{\overline{\mathrm{C}}} \rightarrow j_{*}^{\prime}\left(j^{\prime}\right)^{*} \mathbb{1}_{\overline{\mathrm{C}}}=j_{*}^{\prime} \mathbb{1}_{\mathrm{C}}$ and $a d j_{2}: j_{!}^{\prime} \mathbb{1}_{\mathrm{C}}=j_{!}^{\prime}\left(j^{\prime}\right)^{*} \mathbb{1}_{\overline{\mathrm{C}}} \rightarrow \mathbb{1}_{\overline{\mathrm{C}}}$ are the adjunction maps. Now $c_{*} a d j_{2}$ and $c_{*} a d j_{1}$ are related by duality: we have

$$
\begin{gathered}
c_{*} j_{!}^{\prime} \mathbb{1}_{\mathrm{C}}=\left(c_{*} j_{*}^{\prime}\left(c \circ j^{\prime}\right)^{!} \mathbb{1}_{\mathbf{S p e c} k}\right)^{*}=\left(c_{*} j_{*}^{\prime}\left(j^{\prime}\right)^{*} c^{!} \mathbb{1}_{\mathbf{S p e c} k}\right)^{*}, \\
c_{*} \mathbb{1}_{\overline{\mathrm{C}}}=\left(c_{*} c^{!} \mathbb{1}_{\mathbf{S p e c} k}\right)^{*},
\end{gathered}
$$


and under these identifications, $c_{*} a d j_{2}$ is dual to the morphism

$$
c_{*} a d j_{1}^{!}: c_{*} c^{!} \mathbb{1}_{\text {Spec } k} \longrightarrow c_{*} j_{*}^{\prime}\left(j^{\prime}\right)^{*} c^{!} \mathbb{1}_{\mathbf{S p e c} k},
$$

where $a d j_{1}^{!}$denotes the adjunction map $c^{!} \mathbb{1}_{\text {Spec } k} \rightarrow j_{*}^{\prime}\left(j^{\prime}\right)^{*} c^{\prime} \mathbb{1}_{\text {Spec } k}$ CD2, Thm. 15.2.4]. Fix an isomorphism $\alpha: c^{\prime} \mathbb{1}_{\text {Spec } k} \longrightarrow \mathbb{1}_{\overline{\mathrm{C}}}\left(d_{\mathrm{C}}\right)\left[2 d_{\mathrm{C}}\right]$; according to [W5, Cor. 3.7], such an isomorphism exists. It is unique up to multiplication by global sections of the constant sheaf $F^{*}$ on $C$. Via $\alpha$, the morphism $c_{*} a d j !$ is identified with

$$
c_{*} a d j_{1}\left(d_{\mathrm{C}}\right)\left[2 d_{\mathrm{C}}\right]: c_{*} \mathbb{1}_{\overline{\mathrm{C}}}\left(d_{\mathrm{C}}\right)\left[2 d_{\mathrm{C}}\right] \longrightarrow c_{*} j_{*}^{\prime}\left(j^{\prime}\right)^{*} \mathbb{1}_{\overline{\mathrm{C}}}\left(d_{\mathrm{C}}\right)\left[2 d_{\mathrm{C}}\right],
$$

and this identification does not depend on the choice of $\alpha$.

To summarize the discussion so far: the morphism $a_{*} m$ equals the composition of $c_{*} a d j_{1}$, preceded by the dual of $c_{*} a d j_{1}\left(d_{\mathrm{C}}\right)\left[2 d_{\mathrm{C}}\right]$.

As for the morphism $u$, observe that it, too, can be factorized:

$$
u=\left(j^{\prime}\right)^{*} \circ j_{*}^{\prime}: M_{g m}(\mathrm{C}) \stackrel{j_{*}^{\prime}}{\longrightarrow} M_{g m}(\overline{\mathrm{C}})=M_{g m}^{c}(\overline{\mathrm{C}}) \stackrel{\left(j^{\prime}\right)^{*}}{\longrightarrow} M_{g m}^{c}(\mathrm{C}),
$$

where we denote by $j_{*}^{\prime}$ and $\left(j^{\prime}\right)^{*}$ the morphisms induced by the open immersion $j^{\prime}$ on the level of $M_{g m}$ and $M_{g m}^{c}$, respectively [Vo, pp. 223-224]. According to [Vo, Thm. 4.3.7 3], the dual of $\left(j^{\prime}\right)^{*}$ is identified with

$$
j_{*}^{\prime}\left(-d_{\mathrm{C}}\right)\left[-2 d_{\mathrm{C}}\right]: M_{g m}(\mathrm{C})\left(-d_{\mathrm{C}}\right)\left[-2 d_{\mathrm{C}}\right] \longrightarrow M_{g m}(\overline{\mathrm{C}})\left(-d_{\mathrm{C}}\right)\left[-2 d_{\mathrm{C}}\right] .
$$

To summarize: the morphism $u$ equals the composition of $j_{*}^{\prime}$, followed by the dual of $j_{*}^{\prime}\left(-d_{\mathrm{C}}\right)\left[-2 d_{\mathrm{C}}\right]$.

To relate $a_{*} m$ and $u$, observe that

$$
M_{g m}(\mathrm{C})=\left(c \circ j^{\prime}\right)_{\sharp} \mathbb{1}_{\mathrm{C}}=c_{\sharp} j_{!}^{\prime} \mathbb{1}_{\mathrm{C}}, M_{g m}(\overline{\mathrm{C}})=c_{\sharp} \mathbb{1}_{\overline{\mathrm{C}}},
$$

and under these identifications, the morphism $j_{*}^{\prime}$ equals $c_{\sharp} a d j_{3}$, where $\operatorname{adj} j_{3}$ : $j_{1}^{\prime} \mathbb{1}_{\mathrm{C}}=j_{1}^{\prime}\left(j^{\prime}\right)^{*} \mathbb{1}_{\overline{\mathrm{C}}} \rightarrow \mathbb{1}_{\overline{\mathrm{C}}}$ is the adjunction CD2, Sect. 1.1.34, Sect. 11.1.2, Cor. 16.1.6]. According to one of the projection formulae

$$
\underline{\operatorname{Hom}}_{V}\left(f_{\sharp} M, N\right)=f_{*} \underline{\operatorname{Hom}}_{T}\left(M, f^{*} N\right)
$$

for $f: T \rightarrow V$ smooth [CD2, Sect. 1.1.33], the morphism $j_{*}^{\prime}=c_{\sharp} a d j_{3}$ is dual to $c_{*} a d j_{1}$. Thus, the dual of $u$ equals $c_{*} a d j_{1}$, preceded by the dual of $c_{*} a d_{j}\left(d_{\mathrm{C}}\right)\left[2 d_{\mathrm{C}}\right]$, i.e., it equals $a_{*} m$.

It remains to show that the identification of $a_{*} m$ and the dual of $u$ is equivariant under $\mathrm{CH}_{d_{\mathrm{C}}}\left(\mathrm{C} \times_{U} \mathrm{C}\right)$. Given that $a_{*} m$ is the value at $\pi_{*} \mathbb{1}_{\mathrm{C}}$ of a natural transformation of functors, and that $\mathrm{CH}_{d_{\mathrm{C}}}\left(\mathrm{C} \times_{U} \mathrm{C}\right) \otimes_{\mathbb{Z}} F$ is identified with $\operatorname{End}_{C H M(U)_{F}}\left(\pi_{*} \mathbb{1}_{\mathrm{C}}\right)$, all one needs to establish is that under our identification, the action of $\mathrm{CH}_{d_{\mathrm{C}}}\left(\mathrm{C} \times_{U} \mathrm{C}\right)$ on $(a \circ j)_{*} \pi_{*} \mathbb{1}_{\mathrm{C}}$ coincides with the action of $\mathrm{CH}_{d_{\mathrm{C}}}\left(\mathrm{C} \times_{U} \mathrm{C}\right)$ on the dual of $M_{g m}(\mathrm{C})$, and likewise for $(a \circ j)_{!} \pi_{*} \mathbb{1}_{\mathrm{C}}=M_{g m}^{c}(\mathrm{C})^{*}$. As before, the second compatibility is dual to the first, up to application of a twist by $d_{\mathrm{C}}$ and a shift by $2 d_{\mathrm{C}}$.

As for the identification $(a \circ j)_{*} \pi_{*} \mathbb{1}_{\mathrm{C}}=c_{*} j_{*}^{\prime} \mathbb{1}_{\mathrm{C}}=M_{g m}(\mathrm{C})^{*}$, it is compatible with the action of finite correspondences $\mathfrak{Z} \in c_{U}(\mathrm{C}, \mathrm{C})$ by the very definition of the category of motivic complexes [CD2, Def. 11.1.1]. It remains to cite 
[L, Lemma 5.18]: every class in $\mathrm{CH}_{d_{\mathrm{C}}}\left(\mathrm{C} \times{ }_{U} \mathrm{C}\right)$ can be represented by a cycle $\mathfrak{Z}$ belonging to $c_{U}(\mathrm{C}, \mathrm{C})$.

q.e.d.

Corollary 3.10. Assume that $\mathbb{A}=\mathbb{B}=$ Spec $k$ for a field $k$ admitting strict resolution of singularities, that the structure morphism $a: X \rightarrow \mathbb{B}$ is proper, and that its restriction $a \circ j$ to $U \subset X$ is smooth. Let $\pi: \mathrm{C} \rightarrow U$ proper and smooth, and assume that $C$ is quasi-projective over $k$. Let $e \in$ $\mathrm{CH}_{d_{\mathrm{C}}}\left(\mathrm{C} \times_{U} \mathrm{C}\right) \otimes_{\mathbb{Z}} F$ an idempotent. Assume that the direct factor $\left(\pi_{*} \mathbb{1}_{\mathrm{C}}\right)^{e}$ of the Chow motive $\pi_{*} \mathbb{1}_{\mathrm{C}} \in C H M(U)_{F}$ lies in $C H M(U)_{F, \partial w \neq 0,1}$.

(a) The e-part $\partial M_{g m}(\mathrm{C})^{e}$ of the boundary motive $\partial M_{g m}(\mathrm{C})$ is without weights -1 and 0 . In particular, $\mathrm{C}$ and e satisfy assumption [W3, Asp. 4.2], and therefore, the e-part of the interior motive of $\mathrm{C}, \operatorname{Gr}_{0} M_{g m}(\mathrm{C})^{e}$ is defined [W3, Def. 4.9].

(b) There is a canonical isomorphism

$$
a_{*} j_{! *}\left(\pi_{*} \mathbb{1}_{\mathrm{C}}\right)^{e} \stackrel{\sim}{\longrightarrow}\left(\mathrm{Gr}_{0} M_{g m}(\mathrm{C})^{e}\right)^{*} .
$$

It is compatible with the factorizations

$$
(a \circ j) !\left(\pi_{*} \mathbb{1}_{\mathrm{C}}\right)^{e} \longrightarrow a_{*} j_{! *}\left(\pi_{*} \mathbb{1}_{\mathrm{C}}\right)^{e} \longrightarrow(a \circ j)_{*}\left(\pi_{*} \mathbb{1}_{\mathrm{C}}\right)^{e}
$$

of $a_{*} m$ and

$$
M_{g m}(\mathrm{C})^{e} \longrightarrow \mathrm{Gr}_{0} M_{g m}(\mathrm{C})^{e} \longrightarrow M_{g m}^{c}(\mathrm{C})^{e}
$$

of $u$ under the identification of Proposition 3.8 .

Remark 3.11. (a) The hypothesis on strict resolution of singularities is (implicitly) used twice (apart from the proof of Proposition 3.8). First, the results from [W3, Sect. 4] were formulated only for such fields. This comes mainly from the fact that at the time when [W3] was written, the existence of the motivic weight structure on $D M_{g m}(k)_{F}$ was only established under that hypothesis. Given the main results from [B2], one can dispose of that restriction on $k$ as far as the weight structure is concerned (recall that our ring of coefficients $F$ is assumed to be a $\mathbb{Q}$-algebra).

Second, and more seriously, the hypothesis is used for the construction of the action of $\mathrm{CH}_{d_{\mathrm{C}}}\left(\mathrm{C} \times_{U} \mathrm{C}\right)$ on the boundary motive $\partial M_{g m}(\mathrm{C})$ [W6, Thm. 2.2], and hence for the very definition of $\partial M_{g m}(\mathrm{C})^{e}$. It seems plausible that the hypothesis can be avoided using the main results from $[\mathrm{K}$, Sect. 5.3], in particular, localization for $M_{g m}^{c} \otimes_{\mathbb{Z}} F$ [K, Prop. 5.3.5], but we have not tried.

Given Corollary 3.10, the reader should obviously feel free to define the $e$-part of the interior motive of $\mathrm{C}$ as $\left(a_{*} j_{!_{*}}\left(\pi_{*} \mathbb{1}_{\mathrm{C}}\right)^{e}\right)^{*}$, in case the field $k$ does not admit strict resolution of singularities.

(b) Recall from [W3, Def. 4.1 (a)] that there is a ring $c_{1,2}(\mathrm{C}, \mathrm{C})$ (of "bi-finite correspondences") acting on the exact triangle

$$
(*) \quad \partial M_{g m}(\mathrm{C}) \longrightarrow M_{g m}(\mathrm{C}) \longrightarrow M_{g m}^{c}(\mathrm{C}) \longrightarrow \partial M_{g m}(\mathrm{C})[1] .
$$


Denote by $\bar{c}_{1,2}(\mathrm{C}, \mathrm{C})$ the quotient of $c_{1,2}(\mathrm{C}, \mathrm{C})$ by the kernel of this action. The algebra $\bar{c}_{1,2}(\mathrm{C}, \mathrm{C}) \otimes_{\mathbb{Z}} F$ is a canonical source of idempotent endomorphisms of $(*)$, and it is for such choices that assumption [W3, Asp. 4.2] was formulated. However, [W3, Asp. 4.2] admits an obvious generalization to arbitrary idempotent endomorphisms $e$ of $(*)$. Similarly, [W3, Def. 4.9] and all results from [W3, Sect. 4] remain valid in the present context, up to modifications of the equivariance statement in [W3, Thm. 4.3] under the centralizer of $e$ in $\bar{c}_{1,2}(\mathrm{C}, \mathrm{C})$, and of the explicit description of the effect of duality on $e$ in [W3, Prop. 4.15] (neither of which will be needed in the sequel).

Proof of Corollary 3.10. According to Proposition 3.8,

$$
a_{*} m:(a \circ j)_{!}\left(\pi_{*} \mathbb{1}_{\mathrm{C}}\right)^{e} \longrightarrow(a \circ j)_{*}\left(\pi_{*} \mathbb{1}_{\mathrm{C}}\right)^{e}
$$

is identified with the dual of

$$
u: M_{g m}(\mathrm{C})^{e} \longrightarrow M_{g m}^{c}(\mathrm{C})^{e}
$$

Any choice of cone of $a_{*} m$ is therefore isomorphic to the shift by [1] of the dual of any choice of cone of $u$. But $a_{*} i_{*} i^{*} j_{*}\left(\pi_{*} \mathbb{1}_{\mathrm{C}}\right)^{e}$ is a cone of $a_{*} m$, while $\partial M_{g m}(\mathrm{C})^{e}$ is the shift by $[-1]$ of a cone of $u$. Thus,

$$
\partial M_{g m}(\mathrm{C})^{e} \cong\left(a_{*} i_{*} i^{*} j_{*}\left(\pi_{*} \mathbb{1}_{\mathrm{C}}\right)^{e}\right)^{*} .
$$

Thanks to our additional assumption on $\left(\pi_{*} \mathbb{1}_{\mathrm{C}}\right)^{e}$, and to Proposition 3.1 (b), the motive $a_{*} i_{*} i^{*} j_{*}\left(\pi_{*} \mathbb{1}_{\mathrm{C}}\right)^{e}$ is without weights 0 and 1 . Part (a) of our claim then follows from the compatibility of the motivic weight structure with duality [W5, Thm. 1.12].

The same argument yields that $\operatorname{Gr}_{0}(a \circ j)_{*}\left(\pi_{*} \mathbb{1}_{\mathrm{C}}\right)^{e}$ and $\mathrm{Gr}_{0} M_{g m}(\mathrm{C})^{e}$ are dual to each other. Part (b) thus follows from Theorem 3.4 (c). q.e.d.

Remark 3.12. An alternative proof could be given by showing that the exact triangle

$$
a_{*} j_{!}\left(\pi_{*} \mathbb{1}_{\mathrm{C}}\right) \stackrel{a_{*} m}{\longrightarrow} a_{*} j_{*}\left(\pi_{*} \mathbb{1}_{\mathrm{C}}\right) \longrightarrow a_{*} i_{*} i^{*} j_{*}\left(\pi_{*} \mathbb{1}_{\mathrm{C}}\right) \longrightarrow a_{*} j_{!}\left(\pi_{*} \mathbb{1}_{\mathrm{C}}\right)[1]
$$

is $\mathrm{CH}_{d_{\mathrm{C}}}\left(\mathrm{C} \times_{U} \mathrm{C}\right)$-equivariantly isomorphic to the dual of the exact triangle

$$
M_{g m}^{c}(\mathrm{C})[-1] \longrightarrow \partial M_{g m}(\mathrm{C}) \longrightarrow M_{g m}(\mathrm{C}) \stackrel{u}{\longrightarrow} M_{g m}^{c}(\mathrm{C}) \text {. }
$$

To establish that latter result, one would apply techniques similar to the ones used in the proofs of [W6, Thm. 2.2 and 2.5].

Remark 3.13. (a) Assume that we are in the setting of Corollary 3.10. In particular, $\mathbb{A}=\mathbb{B}=\operatorname{Spec} k$, and the (structure) morphism $a: X \rightarrow \mathbb{B}$ is proper. From Corollary [3.10 (b), and from [W3, Theorems 4.7 and 4.8], it follows that the Chow motive $a_{*} j_{!_{*}}\left(\pi_{*} \mathbb{1}_{\mathrm{C}}\right)^{e}$, i.e., the $e$-part of the intersection motive of $U$ relative to $X$ with coefficients in $\pi_{*} \mathbb{1}_{\mathrm{C}}$, realizes to give the $e$-part of interior cohomology of $C$.

(b) In fact, as is shown in [loc. cit.], the statement from (a) follows from 
the more precise fact that the values of the respective cohomological (Hodge theoretic or $\ell$-adic) realization $\left(H^{m} \circ R\right)_{m \in \mathbb{Z}}$ on the canonical morphisms

$$
(a \circ j)_{!}\left(\pi_{*} \mathbb{1}_{\mathrm{C}}\right)^{e} \longrightarrow a_{*} j_{! *}\left(\pi_{*} \mathbb{1}_{\mathrm{C}}\right)^{e}
$$

and

$$
a_{*} j_{! *}\left(\pi_{*} \mathbb{1}_{\mathrm{C}}\right)^{e} \longrightarrow(a \circ j)_{*}\left(\pi_{*} \mathbb{1}_{\mathrm{C}}\right)^{e}
$$

identify $H^{m} \circ R\left(a_{*} j_{! *}\left(\pi_{*} \mathbb{1}_{\mathrm{C}}\right)^{e}\right)$ with the part of weight $m$ of $H^{m} \circ R((a \circ$ $\left.j)_{!}\left(\pi_{*} \mathbb{1}_{\mathrm{C}}\right)^{e}\right)$, and of $H^{m} \circ R\left((a \circ j)_{*}\left(\pi_{*} \mathbb{1}_{\mathrm{C}}\right)^{e}\right)$, respectively.

(c) The statement from (b) can be shown without using Corollary 3.10 , i.e., without any reference to the interior motive, and for arbitrary objects $M_{U}$ of $C H M(U)_{F, \partial w \neq 0,1}$ instead of $\left(\pi_{*} \mathbb{1}_{\mathrm{C}}\right)^{e}$, by formally imitating the proofs of W3, Theorems 4.7 and 4.8]. The latter make essential use of the existence of weights on the level of realizations; indeed, [W3, Theorems 4.7 and 4.8] should be seen as sheaf theoretic phenomena: for any (Hodge theoretic or $\ell$-adic) sheaf $N_{U}$ which is pure of weight $n$, and such that $i^{*} j_{*} N_{U}$ is without weights $n$ and $n+1$, intersection and interior cohomology with coefficients in $N_{U}$ coincide. In particular, if $M_{U} \in C H M(U)_{F, \partial w \neq 0,1}$ is of Abelian type, then according to [W9, Thm. 7.2], the natural map from intersection cohomology to cohomology identifies intersection cohomology and interior cohomology with coefficients in the realization of $M_{U}$.

(d) For the $\ell$-adic realization, a relative version of statement (c) holds, provided that morphisms in the image of the realization are strict with respect to the weight filtration: the morphism $a: X \rightarrow \mathbb{A}$ is still assumed to be proper, but $\mathbb{A}$ may be different from the base scheme, and the latter need not be a field. For a detailed study of the condition on strictness, we refer to [B3, Sect. 2]; note that it is satisfied in the situation we are about to study in Section 4 .

(e) An analogue of (d) should hold for the Hodge theoretic realization.

The following general result will be used in Section 5.

Proposition 3.14. Let $g: W^{\prime} \rightarrow W$ be a finite, étale morphism of schemes. Then the direct image $g_{*}: D M_{\mathrm{E}, c}\left(W^{\prime}\right)_{F} \rightarrow D M_{\mathrm{B}, c}(W)_{F}$ is weight conservative, i.e., it detects weights. More precisely, let $N^{\prime} \in D M_{\mathrm{E}, c}\left(W^{\prime}\right)_{F}$, and $\alpha \leq \beta$ two integers.

(a) $N^{\prime}$ lies in the heart $C H M\left(W^{\prime}\right)_{F}$ if and only if $g_{*} N^{\prime}$ lies in the heart $C H M(W)_{F}$.

(b) $N^{\prime}$ lies in $D M_{\mathrm{E}, c}\left(W^{\prime}\right)_{F, w \leq \alpha}$ if and only if $g_{*} N^{\prime}$ lies in $D M_{\mathrm{E}, c}(W)_{F, w \leq \alpha}$.

(c) $N^{\prime}$ lies in $D M_{\mathrm{E}, c}\left(W^{\prime}\right)_{F, w \geq \beta}$ if and only if $g_{*} N^{\prime}$ lies in $D M_{\mathrm{b}, c}(W)_{F, w \geq \beta}$.

(d) $N^{\prime}$ is without weights $\alpha, \alpha+1, \ldots, \beta$ if and only if $g_{*} N^{\prime}$ is without weights $\alpha, \alpha+1, \ldots, \beta$. 
Proof. Since $g$ is finite and étale, both $g^{*}$ and $g_{*}$ are weight exact [H, Thm. 3.8 (ii'), (i), (i')]. In particular, the "only if" parts of statements (a) $-(\mathrm{d})$ are true.

As for the "if" parts, note first that $g^{*}=g^{\text {! }}$ [CD2, Thm. 2.4.50 (3), Def. 2.4.12 (2)] and $g_{!}=g_{*}$ (since $g$ is proper). Therefore, there are adjunction morphisms between $\operatorname{id}_{D M_{\mathrm{b}, c}\left(W^{\prime}\right)_{F}}$ and $g^{*} g_{*}$ in both directions. Next, for any $N^{\prime} \in D M_{\mathrm{L}, c}\left(W^{\prime}\right)_{F}$, the composition of the adjunctions

$$
N^{\prime} \longrightarrow g^{*} g_{*} N^{\prime} \longrightarrow N^{\prime}
$$

allows to identify $N^{\prime}$ with a direct factor of $g^{*} g_{*} N^{\prime}$. Statements (a)-(c) thus follow from the fact that the categories $C H M\left(W^{\prime}\right)_{F}, D M_{\mathrm{E}, c}\left(W^{\prime}\right)_{F, w \leq \alpha}$ and $D M_{\mathrm{E}, c}\left(W^{\prime}\right)_{F, w \geq \beta}$ are all pseudo-Abelian. Statement (d) is a consequence of functoriality of weight filtrations avoiding weights $\alpha, \alpha+1, \ldots, \beta$ W3. Prop. 1.7], applied to an idempotent endomorphism of $g^{*} g_{*} N^{\prime}$ cutting out $N^{\prime}$

q.e.d.

Remark 3.15. The analogue of Proposition 3.14 holds for the inverse image $g^{*}$ under a finite, étale morphism $g$, with the same proof, provided that $g$ is surjective. This fact will not be needed in the sequel.

Corollary 3.16. Assume that $\mathbb{A}=\mathbb{B}=$ Spec $k$ for a field $k$ admitting strict resolution of singularities, that the structure morphism $a: X \rightarrow \mathbb{B}$ is proper, and that its restriction $a \circ j$ to $U \subset X$ is smooth. Let $\pi: \mathrm{C} \rightarrow U$ proper and smooth, and assume that $C$ is quasi-projective over $k$. Let $e \in$ $\mathrm{CH}_{d_{\mathrm{C}}}\left(\mathrm{C} \times_{U} \mathrm{C}\right) \otimes_{\mathbb{Z}} F$ an idempotent. Assume that the restriction $a \circ i$ of the structure morphism to $Z \subset X$ is finite. Let $\alpha \leq \beta$ be two integers. Then the following are equivalent.

(1) The motive $i^{*} j_{*}\left(\pi_{*} \mathbb{1}_{\mathrm{C}}\right)^{e} \in D M_{\mathrm{E}, c}(Z)_{F}$ is without weights $\alpha, \alpha+1, \ldots, \beta$.

(2) The motive $\partial M_{g m}(\mathrm{C})^{e} \in D M_{\mathrm{E}, c}(\operatorname{Spec} k)_{F}$ is without weights $-\beta,-(\beta-$ $1), \ldots,-\alpha$.

In particular, the Chow motive $\left(\pi_{*} \mathbb{1}_{\mathrm{C}}\right)^{e}$ lies in $C H M(U)_{F, \partial w \neq 0,1}$ if and only if $\partial M_{g m}(\mathrm{C})^{e}$ is without weights -1 and 0 .

Proof. The field $k$ is perfect; therefore, the reduced scheme $Z_{\text {red }}$ underlying $Z$ is finite and étale over Spec $k$. Given localization for the inclusion of $Z_{\text {red }}$ into $Z$ [CD3, Sect. 2.3], we may thus assume that $g:=a \circ i$ is finite and étale. Given that

$$
\partial M_{g m}(\mathrm{C})^{e} \cong\left(g_{*} i^{*} j_{*}\left(\pi_{*} \mathbb{1}_{\mathrm{C}}\right)^{e}\right)^{*} .
$$

(see the proof of Corollary 3.10), our claim follows from Proposition 3.14. 


\section{A criterion on absence of weights in the boundary}

We keep the geometrical situation of the preceding section: $X$ is a scheme, and $j: U \hookrightarrow X$ an open immersion with complement $i: Z \hookrightarrow X$. For a finite stratification by nilregular locally closed sub-schemes $Z_{\varphi}$ of $Z$, indexed by $\varphi \in \Phi$, recall the definition of the category $D M_{\mathrm{E}, c, \Phi}^{A b}(Z)_{F}$ of $\Phi$-constructible motives of Abelian type over $Z$ [W10, Def. 3.5 (b)]: it is the strict, full, dense, $F$-linear triangulated sub-category of $D M_{\mathrm{B}, c}(Z)_{F}$ generated by the images under $\pi_{*}$ of the objects of $D_{\mathfrak{S}}(S(\mathfrak{S}))_{F}$, the category of $\mathfrak{S}$-constructible Tate motives over $S(\mathfrak{S})$ [W10, Def. 3.3], where

$$
\pi: S(\mathfrak{S}) \longrightarrow Z=Z(\Phi)
$$

runs through the morphisms of Abelian type with target equal to $Z=Z(\Phi)$. According to [W10, Def. 3.5 (a)], this means that $\mathfrak{S}$ is a finite stratification of $S=S(\mathfrak{S})$ by nilregular locally closed sub-schemes, that $\pi$ is a morphism of good stratifications [W10, Def. 3.4], that $\pi$ is proper, and that the following conditions are satisfied.

(1) For any immersion $i_{\tau}: S_{\tau} \hookrightarrow \overline{S_{\sigma}}$ of a stratum $S_{\tau}$ into the closure $\overline{S_{\sigma}}$ of a stratum $S_{\sigma}$, the functor $i_{\tau}^{!}$maps $\mathbb{1}_{\overline{S_{\sigma}}}$ to a Tate motive over $S_{\tau}$.

(2) For all $\sigma \in \mathfrak{S}$ such that $S_{\sigma}$ is a stratum of $\pi^{-1}\left(Z_{\varphi}\right)$, the morphism $\pi_{\sigma}: S_{\sigma} \rightarrow Z_{\varphi}$ can be factorized,

$$
\pi_{\sigma}=\pi_{\sigma}^{\prime} \circ \pi_{\sigma}^{\prime \prime}: S_{\sigma} \stackrel{\pi_{\sigma}^{\prime \prime}}{\longrightarrow} B_{\sigma} \stackrel{\pi_{\sigma}^{\prime}}{\longrightarrow} Z_{\varphi}
$$

such that the motive

$$
\pi_{\sigma, *}^{\prime \prime} \mathbb{1}_{S_{\sigma}} \in D M_{\mathrm{E}, c}\left(B_{\sigma}\right)_{F}
$$

belongs to the category $D M T\left(B_{\sigma}\right)_{F}$ of Tate motives over $B_{\sigma}$, the morphism $\pi_{\sigma}^{\prime}$ is proper and smooth, and its pull-back to any geometric point of $Z_{\varphi}$ lying over a generic point is isomorphic to a finite disjoint union of Abelian varieties.

Definition 4.1. An object $M \in D M_{\mathrm{E}, c}(Z)_{F}$ is a motive of Abelian type over $Z$ if it belongs to the sub-category $D M_{\mathrm{b}, c, \Phi}^{A b}(Z)_{F}$, for a suitable finite stratification $\Phi$ by nilregular locally closed sub-schemes of $Z$. In this situation, we say that $\Phi$ is adapted to $M$.

Let us now fix a generic point Spec $k$ of the base $\mathbb{B}$. For any scheme $Y$, denote by $R_{\ell, Y}$ the (generic) $\ell$-adic realization [W10, Sect. 4]. Its target is the $F$-linear version $D_{c}^{b}\left(Y_{k}\right)_{F}$ of the bounded "derived category" $D_{c}^{b}\left(Y_{k}\right)[\mathrm{E}$, Sect. 6] of constructible $\mathbb{Q}_{\ell}$-sheaves on the fibre $Y_{k}$ of $Y$ over Spec $k \hookrightarrow \mathbb{B}$. According to [CD3, Thm. 7.2.24], the $R_{\ell, \bullet}$ are compatible with the functors $f^{*}, f_{*}, f_{!}, f^{!}$. Furthermore, they are symmetric monoidal; in particular,

$$
R_{\ell, Y}\left(\mathbb{1}_{Y}\right)=\mathbb{Q}_{\ell, Y_{k}},
$$


where $\mathbb{Q}_{\ell, Y_{k}}$ denotes the $\ell$-adic structure sheaf on $Y_{k}$.

The following is an immediate consequence of the main result from [W10].

Theorem 4.2. Assume that $k$ is of characteristic zero. Let $\ell$ be a prime. Let $N \in D M_{\mathrm{E}, c, \Phi}^{A b}(Z)_{F}$. Assume that the generic points of all strata $Z_{\varphi}$, $\varphi \in \Phi$, lie over $\operatorname{Spec} k \hookrightarrow \mathbb{B}$. For $\varphi \in \Phi$, denote by $i_{\varphi}$ the immersion of $Z_{\varphi}$ into $Z$.

(a) Let $\alpha \in \mathbb{Z}$. Then $N$ lies in $D M_{\mathrm{Б}, c, \Phi}^{A b}(Z)_{F, w \leq \alpha}$ if and only if for all $n \in \mathbb{Z}$, and all $\varphi \in \Phi$, the perverse cohomology sheaf

is of weights $\leq n+\alpha$.

$$
H^{n} i_{\varphi}^{*} R_{\ell, Z}(N)
$$

(b) Let $\beta \in \mathbb{Z}$. Then $N$ lies in $D M_{\mathrm{E}, c, \Phi}^{A b}(Z)_{F, w \geq \beta}$ if and only if for all $n \in \mathbb{Z}$, and all $\varphi \in \Phi$, the perverse cohomology sheaf

$$
H^{n} i_{\varphi}^{!} R_{\ell, Z}(N)
$$

is of weights $\geq n+\beta$.

Remark 4.3. (a) The conditions on the generic points of the strata $Z_{\varphi}$ are empty when $\mathbb{B}$ is itself the spectrum of a field of characteristic zero.

(b) Recall from [B1, Prop. 2.1.2 1] that any additive functor $\mathcal{H}$ from a triangulated category $\mathcal{C}$ carrying a weight structure $w$, to an Abelian category $\mathfrak{A}$ admits a canonical weight filtration by sub-functors

$$
\ldots \subset W_{n} \mathcal{H} \subset W_{n+1} \mathcal{H} \subset \ldots \subset \mathcal{H} .
$$

For any $m \in \mathbb{Z}$, one defines

$$
\mathcal{H}^{m}: \mathcal{C} \longrightarrow \mathfrak{A}, M \longrightarrow \mathcal{H}(X[m]) ;
$$

according to the usual convention, the weight filtration of $\mathcal{H}^{m}(M)$ equals the weight filtration of $\mathcal{H}(X[m])$, i.e., it differs by décalage from the intrinsic weight filtration of the covariant additive functor $\mathcal{H}^{m}$.

If $k$ is finitely generated over $\mathbb{Q}$, then there is an instrinsic notion of weights on those perverse sheaves on $Y_{k}$, which are in the image of the cohomological realization [B3, Prop. 2.5.1 (II)].

In general, the weights of $H^{*} R_{\ell, Y}(M)$ are by definition those induced by the weight filtration of the functor $H^{*} R_{\ell, Y}$ (these coincide with the above when $k$ is finitely generated over $\mathbb{Q}$ ).

Proof of Theorem 4.2. The motive $N$ belongs to $D M_{\mathrm{E}, c, \Phi}^{A b}(Z)_{F, w \leq \alpha}$ if and only if for all $\varphi \in \Phi$,

$$
i_{\varphi}^{*} N \in D M_{\mathrm{E}, c, \Phi}^{A b}\left(Z_{\varphi}\right)_{F, w \leq \alpha} .
$$

According to [W10, Thm. $4.4(\mathrm{~b})]$, the latter condition is equivalent to

$$
H^{n} R_{\ell, Z_{\varphi}}\left(i_{\varphi}^{*} N\right) \text { is of weights } \leq n+\alpha,
$$


for all $n \in \mathbb{Z}$. But thanks to the compatibility of $R_{\ell, \bullet}$ with $i_{\varphi}^{*}$, we have

$$
R_{\ell, Z_{\varphi}}\left(i_{\varphi}^{*} N\right)=i_{\varphi}^{*} R_{\ell, Z}(N) .
$$

This proves part (a) of our claim. Dualizing, we obtain the proof of part (b).

q.e.d.

Together with one of the main compatibility results from [W9], we obtain the following.

Theorem 4.4. Assume that $k$ is of characteristic zero. Let $\ell$ be a prime. Let $M_{U} \in C H M(U)_{F}$, such that $R_{\ell, U}\left(M_{U}\right)$ is concentrated in a single perverse degree, and such that $i^{*} j_{*} M_{U} \in D M_{\mathrm{b}, c}(Z)_{F}$ is of Abelian type. Let $\Phi$ a stratification of $Z$ adapted to $i^{*} j_{*} M_{U}$. Assume that the generic points of all $Z_{\varphi}, \varphi \in \Phi$, lie over $\operatorname{Spec} k \hookrightarrow \mathbb{B}$. For $\varphi \in \Phi$, denote by $i_{\varphi}$ the immersion of $Z_{\varphi}$ into $Z$.

(a) The motive $M_{U}$ belongs to $C H M(U)_{F, \partial w \neq 0,1}$ if and only if for all $n \in \mathbb{Z}$, and all $\varphi \in \Phi$, the following conditions hold: the perverse cohomology sheaf

$$
H^{n} i_{\varphi}^{*} i^{*} j_{! *} R_{\ell, U}\left(M_{U}\right)
$$

is of weights $\leq n-1$, and

$$
H^{n} i_{\varphi}^{!} i^{!} j_{!_{*}} R_{\ell, U}\left(M_{U}\right)
$$

is of weights $\geq n+1$. In particular, the intermediate extension $j_{\text {!* }} M_{U}$ is then defined up to unique isomorphism, as a Chow motive over $X$.

(b) Let $\alpha \leq 0$ and $\beta \geq 1$ two integers. The motive $i^{*} j_{*} M_{U}$ is without weights $\alpha, \alpha+1, \ldots, \beta$ if and only if for all $n \in \mathbb{Z}$, and all $\varphi \in \Phi$, the following conditions hold: the perverse cohomology sheaf

$$
H^{n} i_{\varphi}^{*} i^{*} j_{! *} R_{\ell, U}\left(M_{U}\right)
$$

is of weights $\leq n+\alpha-1$, and

$$
H^{n} i_{\varphi}^{!} i^{!} j_{! *} R_{\ell, U}\left(M_{U}\right)
$$

is of weights $\geq n+\beta$.

(c) Let $\alpha \leq 0$ and $\beta \geq 1$ two integers. Assume that for all $n \in \mathbb{Z}$, and all $\varphi \in \Phi$, the following conditions hold: the perverse cohomology sheaf

$$
H^{n} i_{\varphi}^{*} i^{*} j_{! *} R_{\ell, U}\left(M_{U}\right)
$$

is of weights $\leq n+\alpha-1$, and

$$
H^{n} i_{\varphi}^{!} i^{!} j_{! *} R_{\ell, U}\left(M_{U}\right)
$$

is of weights $\geq n+\beta$. Then $a_{*} j_{!} M_{U}$ is without weights $\alpha-1, \alpha, \ldots,-1$, and $a_{*} j_{*} M_{U}$ is without weights $1,2, \ldots, \beta$, for any proper morphism $a: X \rightarrow \mathbb{A}$.

By slight abuse of notation, we write $j_{! *} R_{\ell, U}\left(M_{U}\right)$ for

$$
\left(j_{!_{*}}\left(R_{\ell, U}\left(M_{U}\right)[s]\right)\right)[-s],
$$


if $R_{\ell, U}\left(M_{U}\right)$ is concentrated in perverse degree $s$.

Proof of Theorem 4.4. Part (a) follows from part (b) (take $\alpha=0$ and $\beta=1$ ), and from Definitions 2.1 (a) and 2.4, while part (c) is implied by (b) and Theorem 3.4 (a) and (b).

As for part (b), note that according to [W10, Thm. 3.10], the heart of the weight structure of $D M_{\mathrm{E}, c, \Phi}^{A b}(Z)_{F}$ is semi-primary. Therefore, Theorem 2.7 can be applied; the motive $i^{*} j_{*} M_{U}$ is thus without weights $\alpha, \alpha+1, \ldots, \beta$ if and only if

$$
i^{*} j_{! *} M_{U} \in \mathcal{C}(Z)_{w \leq \alpha-1} \quad \text { and } \quad i^{!} j_{! *} M_{U} \in \mathcal{C}(Z)_{w \geq \beta} .
$$

By Theorem 4.2, this is in turn equivalent to the following: for all $n \in \mathbb{Z}$, and all $\varphi \in \Phi$,

$$
H^{n} i_{\varphi}^{*} R_{\ell, Z}\left(i^{*} j_{! *} M_{U}\right)
$$

is of weights $\leq n+\alpha-1$, and

$$
H^{n} i_{\varphi}^{!} R_{\ell, Z}\left(i^{*} j_{! *} M_{U}\right)
$$

is of weights $\geq n+\beta$. Thanks to compatibility of $R_{\ell, \bullet}$ with $i^{*}$ and $i^{!}$, we have

$$
i^{*} \circ R_{\ell, X}=R_{\ell, Z} \circ i^{*} \text { and } R_{\ell, Z} \circ i^{!}=i^{!} \circ R_{\ell, X} .
$$

The compatibility of $R_{\ell, \bullet}$ with $j_{! *}$ is the content of [W9, Thm. $7.2(\mathrm{~b})$ ].

q.e.d.

Remark 4.5. (a) Given the full, triangulated sub-category $D M_{\mathrm{b}, c, \Phi}^{A b}(Z)_{F}$ of $D M_{\mathrm{L}, c}(Z)_{F}$, there is first a maximal choice of full, triangulated subcategory $\mathcal{D}(U)$ of $D M_{\mathrm{Б}, c}(U)_{F}$, which glues with $D M_{\mathrm{Б}, c, \Phi}^{A b}(Z)_{F}$, to give a full, triangulated sub-category of $D M_{\mathrm{L}, c}(X)_{F}$, namely the full sub-category of objects $M_{U}$ satisfying $i^{*} j_{*} M_{U} \in D M_{\mathrm{E}, c, \Phi}^{A b}(Z)_{F}$ (cmp. [W9, Prop 4.1]). Inside $\mathcal{D}(U)$, we then find the maximal choice of full, triangulated sub-category $\mathcal{C}(U)$ of $D M_{\mathrm{E}, c}(U)_{F}$, which glues with $D M_{\mathrm{E}, c, \Phi}^{A b}(Z)_{F}$, to give a full, triangulated sub-category $\mathcal{C}(X)$ of $D M_{\mathrm{E}, c}(X)_{F}$, and which in addition inherits a weight structure from the motivic weight structure on $D M_{\mathrm{L}, c}(U)_{F}$, namely the full triangulated sub-category generated by objects $M_{U}$ of $C H M(U)_{F}$ satisfying $i^{*} j_{*} M_{U} \in D M_{\mathrm{E}, c, \Phi}^{A b}(Z)_{F}$. The theory from [W9, Sect. 2] and from the present Section 2 can thus be applied to the triplet of categories $\mathcal{C}(U)$, $\mathcal{C}(X) \subset D M_{\mathrm{L}, c}(X)_{F}$, and $\mathcal{C}(Z)=D M_{\mathrm{E}, c, \Phi}^{A b}(Z)_{F}$.

(b) In particular, if an object $M_{U}$ of $C H M(U)_{F}$ is such that $i^{*} j_{*} M_{U} \in$ $D M_{\mathrm{b}, c, \Phi}^{A b}(Z)_{F}$, i.e., $M_{U}$ belongs to $\mathcal{C}(U)_{w=0}$, where $\mathcal{C}(U)$ is as in (a), then $\left(j_{! *} M_{U}\right.$ exists, and $i^{*} j_{! *} M_{U}$ and $i^{!} j_{! *} M_{U}$ belong to $D M_{\mathrm{L}, c, \Phi}^{A b}(Z)_{F}$. This fact was implicitly used in the proof of Theorem 4.4.

(c) A priori, the application of [W9, Thm. 7.2] necessitates the validity of [W9, Asp. 7.1]: the motive $M_{U}$ belongs to the triangulated sub-category $\pi_{*} D M T_{\mathfrak{S}_{U}}\left(S\left(\mathfrak{S}_{U}\right)\right)_{F, w=0}^{\natural}$ of $C H M(U)_{F}$, for an extension of $\pi: S(\mathfrak{S}) \rightarrow Z$ to $X$. While [W9, Asp. 7.1 (b), (c)] belong to the hypotheses of Theorem 4.4, 
W9, Asp. 7.1 (a)] is replaced by the condition that $M_{U}$ belong to the category $\mathcal{C}(U)$ from (a). The proof of [W9, Thm. $7.2(\mathrm{~b})]$ carries over to this more general context without any modification.

Corollary 4.6. Assume that $k$ is of characteristic zero. Let $\ell$ be a prime. Let $M_{U} \in C H M(U)_{F}$, such that $R_{\ell, U}\left(M_{U}\right)$ is concentrated in a single perverse degree $s$, and such that $R_{\ell, U}\left(M_{U}\right)$ is auto-dual up to a shift and a twist:

$$
\mathbb{D}_{\ell, U}\left(R_{\ell, U}\left(M_{U}\right)\right) \cong R_{\ell, U}\left(M_{U}\right)(s)[2 s]
$$

$\left(\mathbb{D}_{\ell, U}=\ell\right.$-adic local duality on $\left.U\right)$. Assume in addition that $i^{*} j_{*} M_{U}$ is of Abelian type. Let $\Phi$ a stratification of $Z$ adapted to $i^{*} j_{*} M_{U}$. Assume that the generic points of all $Z_{\varphi}, \varphi \in \Phi$, lie over $\operatorname{Spec} k \hookrightarrow \mathbb{B}$. For $\varphi \in \Phi$, denote by $i_{\varphi}$ the immersion of $Z_{\varphi}$ into $Z$.

(a) The motive $M_{U}$ belongs to $C H M(U)_{F, \partial w \neq 0,1}$ if and only if for all $n \in \mathbb{Z}$, and all $\varphi \in \Phi$, the following holds: the perverse cohomology sheaf

$$
H^{n} i_{\varphi}^{*} i^{*} j_{! *} R_{\ell, U}\left(M_{U}\right)
$$

is of weights $\leq n-1$.

(b) Let $\beta \geq 1$ be an integer. The following are equivalent.

(b1) The motive $i^{*} j_{*} M_{U}$ is without weights $-\beta+1,-\beta+2, \ldots, \beta$.

(b2) For all $n \in \mathbb{Z}$, and all $\varphi \in \Phi$,

$$
H^{n} i_{\varphi}^{*} i^{*} j_{! *} R_{\ell, U}\left(M_{U}\right)
$$

is of weights $\leq n-\beta$.

(b3) For all $n \in \mathbb{Z}$, and all $\varphi \in \Phi$,

$$
H^{n} i_{\varphi}^{!} i^{!} j_{! *} R_{\ell, U}\left(M_{U}\right)
$$

is of weights $\geq n+\beta$.

(c) Let $\beta \geq 1$ be an integer, and assume that one of the equivalent conditions (b1), (b2), (b3) is satisfied. Then $a_{*} j_{!} M_{U}$ is without weights $-\beta,-\beta+$ $1, \ldots,-1$, and $a_{*} j_{*} M_{U}$ is without weights $1,2, \ldots, \beta$, for any proper morphism $a: X \rightarrow \mathbb{A}$.

Proof. Part (a) is a special case of part (b) (take $\beta=1$, and use the equivalence (b1) $\Leftrightarrow$ (b2)). Similarly, part (c) follows from (b2) $\Leftrightarrow$ (b3), and from Theorem 4.4 (c) (with $\alpha=-\beta+1$ ).

As for part (b), observe that for all $n \in \mathbb{Z}$, and all $\varphi \in \Phi$,

$$
H^{n} i_{\varphi}^{!} i^{!} j_{! *} R_{\ell, U}\left(M_{U}\right)
$$

is dual to

$$
H^{-n} i_{\varphi}^{*} i^{*} j_{! *} \mathbb{D}_{\ell, U}\left(R_{\ell, U}\left(M_{U}\right)\right)
$$


By assumption, weight $w$ occurs in

$$
H^{-n} i_{\varphi}^{*} i^{*} j_{! *} \mathbb{D}_{\ell, U}\left(R_{\ell, U}\left(M_{U}\right)\right) \cong H^{-n} i_{\varphi}^{*} i^{*} j_{! *}\left(R_{\ell, U}\left(M_{U}\right)(s)[2 s]\right)
$$

if and only if weight $2 s+w$ occurs in

$$
H^{2 s-n} i_{\varphi}^{*} i^{*} j_{! *} R_{\ell, U}\left(M_{U}\right)
$$

Therefore, conditions (b2) and (b3) are equivalent to each other. According to Theorem 4.4 (b) (with $\alpha=-\beta+1$ ), each of them is thus equivalent to (b1).

q.e.d.

Remark 4.7. Applying the variant of the theory from Section 2 sketched in Remark 2.8, we see that in Corollary 4.6 (b), conditions (b1)-(b3) are also equivalent to

(b4) The motive $i^{*} j_{*} M_{U}$ is without weights $-\beta+1,-\beta+2, \ldots, 0$.

(b5) The motive $i^{*} j_{*} M_{U}$ is without weights $1,2, \ldots, \beta$.

Clearly condition (b1) implies both (b4) and (b5). We claim that (b4) implies (b2), and that (b5) implies (b3). Indeed, according to Remark 2.8 (b), condition (b4) implies

$$
i^{*} j_{! *} M_{U} \in \mathcal{C}(Z)_{w \leq-\beta}
$$

while condition (b5) implies

$$
i^{!} j_{! *} M_{U} \in \mathcal{C}(Z)_{w \geq \beta}
$$

Now apply Theorem 4.2 (a) and (b).

Together with the comparison result from Section 3 , we get the following.

Theorem 4.8. Assume that $\mathbb{B}=\operatorname{Spec} k$ for a field $k$ of characteristic zero, that the structure morphism $a: X \rightarrow \mathbb{B}$ is proper, and that its restriction $a \circ j$ to $U \subset X$ is smooth. Let $\pi: \mathrm{C} \rightarrow U$ proper and smooth, and assume that $C$ is quasi-projective over $k$. Let $e \in \mathrm{CH}_{d_{\mathrm{C}}}\left(\mathrm{C} \times_{U} \mathrm{C}\right) \otimes_{\mathbb{Z}} F$ an idempotent. Let $\ell$ be a prime. Assume that $R_{\ell, U}\left(\pi_{*} \mathbb{1}_{\mathrm{C}}\right)^{e}$ is concentrated in a single perverse degree $s$, and that $R_{\ell, U}\left(\pi_{*} \mathbb{1}_{\mathrm{C}}\right)^{e}$ is auto-dual up to a shift and a twist:

$$
\mathbb{D}_{\ell, U}\left(R_{\ell, U}\left(\pi_{*} \mathbb{1}_{\mathrm{C}}\right)^{e}\right) \cong R_{\ell, U}\left(\pi_{*} \mathbb{1}_{\mathrm{C}}\right)^{e}(s)[2 s] .
$$

Assume in addition that the motive $i^{*} j_{*}\left(\pi_{*} \mathbb{1}_{\mathrm{C}}\right)^{e}$ is of Abelian type. Let $\Phi$ a stratification of $Z$ adapted to $i^{*} j_{*}\left(\pi_{*} \mathbb{1}_{\mathrm{C}}\right)^{e}$. For $\varphi \in \Phi$, denote by $i_{\varphi}$ the immersion of $Z_{\varphi}$ into $Z$.

(a) If for all $n \in \mathbb{Z}$, and all $\varphi \in \Phi$, the perverse cohomology sheaf

$$
H^{n} i_{\varphi}^{*} i^{*} j_{! *} R_{\ell, U}\left(\pi_{*} \mathbb{1}_{\mathrm{C}}\right)^{e}
$$

is of weights $\leq n-1$, then $\partial M_{g m}(\mathrm{C})^{e}$ is without weights -1 and 0 . 
(b) If for all $n \in \mathbb{Z}$, and all $\varphi \in \Phi$, the perverse cohomology sheaf

$$
H^{n} i_{\varphi}^{*} i^{*} j_{!_{*}} R_{\ell, U}\left(\pi_{*} \mathbb{1}_{\mathrm{C}}\right)^{e}
$$

is of weights $\leq n-1$, then there is a canonical isomorphism

$$
a_{*} j_{! *}\left(\pi_{*} \mathbb{1}_{\mathrm{C}}\right)^{e} \stackrel{\sim}{\longrightarrow}\left(\mathrm{Gr}_{0} M_{g m}(\mathrm{C})^{e}\right)^{*} .
$$

Proof. Combine Corollaries 4.6 and 3.10.

q.e.d.

Remark 4.9. The condition on auto-duality of $R_{\ell, U}\left(\pi_{*} \mathbb{1}_{\mathrm{C}}\right)^{e}$ is satisfied if the cycle $e$ and its transposition ${ }^{t} e$ have identical images under $R_{\ell, C}$.

\section{Examples: the boundary of certain Shimu- ra varieties}

The common features of the examples to be reviewed in the present section are the following. The open immersion $j$ equals the inclusion of a (pure) Shimura variety of $P E L$-type $M^{K}$, whose level $K$ is neat, into its Baily-Borel compactification $\left(M^{K}\right)^{*}$. The complement $i$ thus equals the closed immersion of the boundary $\partial\left(M^{K}\right)^{*}$ of $\left(M^{K}\right)^{*}$. The variety $M^{K}$ is associated to (pure) Shimura data $(G, \mathcal{H})$; in particular, $G$ is a connected reductive group over $\mathbb{Q}$. The finite stratification $\Phi$ of $\partial\left(M^{K}\right)^{*}$ is indexed by the $G(\mathbb{Q})$-conjugation classes of rational boundary components of $(G, \mathcal{H})$. Each stratum is a finite disjoint union of locally closed sub-varieties of $\partial\left(M^{K}\right)^{*}$, each of which is a quotient by the action of a finite group of a (pure) Shimura variety associated to Shimura data, which are "smaller" than $(G, \mathcal{H})$. All strata are nilregular. The category $D M_{\mathrm{b}, c, \Phi}^{A b}\left(\partial\left(M^{K}\right)^{*}\right)_{F}$ of $\Phi$-constructible motives of Abelian type over $\partial\left(M^{K}\right)^{*}$ is therefore defined.

As for the source of the relative Chow motives in $C H M\left(M^{K}\right)_{F}$, note that the Shimura data $(G, \mathcal{H})$ being of $P E L$-type, there is on the one hand a canonical faithful representation $V$ of $G$ (the latter being defined as the group of endomorphisms of $V$ commuting with a certain semi-simple algebra, and respecting, up to scalars, a certain anti-symmetric bilinear form on $V$ ). On the other hand, given the modular interpretation of $M^{K}$, there is a universal Abelian scheme $\pi: \mathrm{B} \rightarrow M^{K}$. Denote by $\pi_{*}^{m} \mathbb{1}_{\mathrm{B}}, m \geq 0$, the $m$-th ChowKünneth component of the Chow motive $\pi_{*} \mathbb{1}_{\mathrm{B}}^{*}$ over $M^{\bar{K}}$ [DM, Thm. 3.1].

Theorem 5.1 ([ Anc, Thm. 8.6]). There is an F-linear tensor functor

$$
\widetilde{\mu}: \operatorname{Rep}\left(G_{F}\right) \longrightarrow C H M^{s}\left(M^{K}\right)_{F}
$$

from the category $\operatorname{Rep}\left(G_{F}\right)$ of algebraic representations of $G$ in $F$-modules of finite type to the full sub-category $C H M^{s}\left(M^{K}\right)_{F}$ of $C H M\left(M^{K}\right)_{F}$ of smooth Chow motives over $M^{K}$. It has the following properties. 
(a) The composition of $\widetilde{\mu}$ with the cohomological Hodge theoretic realization is isomorphic to the canonical construction functor $\mu_{\mathbf{H}}$ (e.g. [W1, Thm. 2.2]) to the category of admissible graded-polarizable variations of Hodge structure on $M_{\mathbb{C}}^{K}$.

(b) The composition of $\widetilde{\mu}$ with the cohomological $\ell$-adic realization is isomorphic to the canonical construction functor $\mu_{\ell}$ (e.g. [W1, Chap. 4]) to the category of lisse $\ell$-adic sheaves on $M^{K}$.

(c) The functor $\widetilde{\mu}$ commutes with Tate twists in the following sense: for any $W \in \operatorname{Rep}\left(G_{F}\right)$ and $n \in \mathbb{Z}$, we have

$$
\widetilde{\mu}(W(n))=\widetilde{\mu}(W)(n)[2 n] .
$$

(d) The functor $\widetilde{\mu}$ maps the representation $V$ to the Chow motive $\pi_{*}^{1} \mathbb{1}_{\mathrm{B}}(1)[2]$ over $M^{K}$.

Proof. Parts (a), (c) and (d) are identical to [Anc, Thm. 8.6]; as for (d), note that the anti-symmetric bilinear form implicit in the $P E L$-data induces an isomorphism between the dual of $V$ and $V(-1)$.

As for part (b), repeat the proof of [loc. cit.], observing that the $\ell$-adic analogue of [Anc, Prop. 8.5] holds (the base change to $\mathbb{Q}_{\ell}$ of the sub-group $G_{1}$ of $G$ coincides with the Lefschetz group).

q.e.d.

Additional common features of Shimura varieties are that the base scheme $\mathbb{B}$ equals $\operatorname{Spec} E$, for a number field $E$ called the reflex field of $(G, \mathcal{H})$, and that the ring of coefficients $F$ is equipped with a canonical structure of $F^{\prime}$ algebra, for a number field $F^{\prime}$ over which $G$ is split.

Definition 5.2. Fix a maximal split torus $T$ of $G_{F^{\prime}}$, and a dominant character $\underline{\alpha}$ of $T$.

(a) Denote by $V_{\underline{\alpha}} \in \operatorname{Rep}\left(G_{F}\right)$ the irreducible representation of highest weight $\underline{\alpha}$.

(b) Define $\underline{\underline{\alpha}} \mathcal{V} \in C H M^{s}\left(M^{K}\right)_{F} \subset C H M\left(M^{K}\right)_{F}$ as

$$
\underline{\alpha} \mathcal{V}:=\widetilde{\mu}\left(V_{\underline{\alpha}}\right) \text {. }
$$

Example 5.3. Our first example concerns modular curves. The reductive group $G$ equals $G L_{2, \mathbb{Q}}$, the reflex field equals $\mathbb{Q}$, meaning that the base scheme $\mathbb{B}$ equals Spec $\mathbb{Q}$, and $F=F^{\prime}=\mathbb{Q}$. The dominant character $\underline{\alpha}$ is identified with a pair of integers $(k, r)$, with $k \geq 0$ and $r \equiv k \bmod 2$ : choosing $T \subset G L_{2, \mathbb{Q}}$ to be equal to the sub-group of diagonal matrices, we have

$$
\underline{\alpha}: T \longrightarrow \mathbb{G}_{m, \mathbb{Q}}, \operatorname{diag}\left(a, a^{-1} q\right) \longmapsto a^{k} q^{-\frac{r+k}{2}} .
$$

The canonical representation $V$ equals the standard two-dimensional representation of $G L_{2, \mathbb{Q}}$. Then,

$$
V_{\underline{\alpha}}=\operatorname{Sym}^{k} V\left(-\frac{r+k}{2}\right) \in \operatorname{Rep}\left(G L_{2, \mathbb{Q}}\right),
$$


where we denote by $\operatorname{Sym}^{k} V$ the $k$-th symmetric power of $V$. Theorem 5.1 therefore shows that

$$
\underline{\alpha} \mathcal{V}=\operatorname{Sym}^{k} \pi_{*}^{1} \mathbb{1}_{\mathrm{B}}\left(\frac{-r+k}{2}\right)[-r+k] \in C H M\left(M^{K}\right)_{\mathbb{Q}},
$$

where $\pi: \mathrm{B} \rightarrow M^{K}$ is the universal elliptic curve.

The level $K$ equals the kernel of the reduction

$$
G L_{2}(\widehat{\mathbb{Z}}) \longrightarrow G L_{2}(\mathbb{Z} / n \mathbb{Z})
$$

for a fixed integer $n \geq 3$.

We claim that the motive $i^{*} j_{*} \underline{\mathcal{V}} \in D M_{\mathrm{E}, c}\left(\partial\left(M^{K}\right)^{*}\right)$ is without weights

$$
-(k-1),-(k-2), \ldots, k,
$$

and that both weights $-k$ and $k+1$ do occur in $i^{*} j_{*} \underline{\alpha}$ V. In particular, $\underline{\alpha} \mathcal{V}$ belongs to $C H M\left(M^{K}\right)_{\mathbb{Q}, \partial w \neq 0,1} \subset C H M\left(M^{K}\right)_{\mathbb{Q}}$ if and only if $k \geq 1$, i.e., if and only if $\underline{\alpha}$ is regular.

In order to show the claim, note first that for fixed $k \geq 0$, its validity does not depend on the value of $r \equiv k \bmod 2$. We may therefore assume that $r=k$, i.e., that

$$
\underline{\alpha} \mathcal{V}=\operatorname{Sym}^{k} \pi_{*}^{1} \mathbb{1}_{\mathrm{B}} .
$$

The Chow motive $\underline{\alpha} \mathcal{V}$ thus equals a direct factor of $\pi_{k, *} \mathbb{1}_{\mathrm{B}^{k}}$, where we denote by

$$
\pi_{k}: \mathrm{B}^{k}:=\mathrm{B} \times_{M^{K}} \ldots \times_{M^{K}} \mathrm{~B} \longrightarrow M^{K}
$$

the projection of the $k$-fold fibre product $\mathrm{B}^{k}$ to $M^{K}$. Concretely, the symmetric group $\mathfrak{S}_{k}$ acts on $\mathrm{B}^{k}$ by permutations, the $k$-th power of the group $\mathbb{Z} / n \mathbb{Z}$ by translations, and the $k$-th power of the group $\mu_{2}$ by inversion in the fibres. Altogether [S, Sect. 1.1.1], this gives a canonical action of the semi-direct product

$$
\Gamma_{k}:=\left((\mathbb{Z} / n \mathbb{Z})^{2} \rtimes \mu_{2}\right)^{k} \rtimes \mathfrak{S}_{k}
$$

by automorphisms on $B^{k}$. As in $\left[\underline{S}\right.$, Sect. 1.1.2], let $\varepsilon: \Gamma_{k} \rightarrow\{ \pm 1\}$ be the morphism which is trivial on $(\mathbb{Z} / n \mathbb{Z})^{2 k}$, is the product map on $\mu_{2}^{k}$, and is the sign character on $\mathfrak{S}_{k}$. Let $e$ denote the idempotent in the group ring $\mathbb{Q}\left[\Gamma_{k}\right]$ associated to $\varepsilon$ :

$$
e:=\frac{1}{\left(2 n^{2}\right)^{k} \cdot k !} \sum_{\gamma \in \Gamma_{k}} \varepsilon(\gamma)^{-1} \cdot \gamma=\frac{1}{\left(2 n^{2}\right)^{k} \cdot k !} \sum_{\gamma \in \Gamma_{k}} \varepsilon(\gamma) \cdot \gamma
$$

By passage to the graph, every endomorphism of the $M^{K}$-scheme $\mathrm{B}^{k}$ yields a cycle on $B^{k} \times{ }_{M^{K}} B^{k}$ of dimension $d_{\mathrm{B}^{k}}=k+1$. Therefore, we may and do consider $e$ as an idempotent of $\mathrm{CH}_{k+1}\left(\mathrm{~B}^{k} \times_{M^{K}} \mathrm{~B}^{k}\right) \otimes_{\mathbb{Z}} \mathbb{Q}$. Then,

$$
\underline{\alpha} \mathcal{V}=\operatorname{Sym}^{k} \pi_{*}^{1} \mathbb{1}_{\mathrm{B}}=\left(\pi_{k, *} \mathbb{1}_{\mathrm{B}^{k}}\right)^{e} .
$$


Next, note that $\partial\left(M^{K}\right)^{*}$, the scheme of cusps of $M^{K}$, is finite over Spec $\mathbb{Q}$. According to Corollary [3.16, our claim is equivalent to the following: the motive $\partial M_{g m}\left(\mathrm{~B}^{k}\right)^{e} \in D M_{\mathrm{L}, c}(\mathbb{Q})_{\mathbb{Q}}$ is without weights $-k,-(k-1), \ldots, k-1$, and both weights $-(k+1)$ and $k$ occur in $\partial M_{g m}\left(\mathrm{~B}^{k}\right)^{e}$.

Independently of everything said in this article, this latter claim can be proved purely geometrically. More precisely, using the detailed analysis of the geometry of the boundary of the canonical compactification of $\mathrm{B}^{k}$ from [S, Sect. 2, 3], one shows that there is an exact triangle

$$
M_{g m}\left(\partial\left(M^{K}\right)^{*}\right)(k+1)[k+1] \longrightarrow \partial M_{g m}\left(\mathrm{~B}^{k}\right)^{e} \longrightarrow M_{g m}\left(\partial\left(M^{K}\right)^{*}\right)[k] \stackrel{[1]}{\longrightarrow}
$$

in $D M_{\mathrm{E}, c}(\mathbb{Q})_{\mathbb{Q}}[\mathrm{W} 3$, Ex. 4.16, Rem. $3.5(\mathrm{~b})]$. But $M_{g m}\left(\partial\left(M^{K}\right)^{*}\right)(k+1)[k+1]$ is pure of weight $-(k+1)$, and $M_{g m}\left(\partial\left(M^{K}\right)^{*}\right)[k]$ is pure of weight $k$.

For $k \geq 1$, this shows that $\partial M_{g m}\left(\mathrm{~B}^{k}\right)^{e}$ is indeed without weights $-k,-(k-$ $1), \ldots, k-1$. In particular,

$$
M_{g m}\left(\partial\left(M^{K}\right)^{*}\right)(k+1)[k+1] \longrightarrow \partial M_{g m}\left(\mathrm{~B}^{k}\right)^{e} \longrightarrow M_{g m}\left(\partial\left(M^{K}\right)^{*}\right)[k] \stackrel{[1]}{\longrightarrow}
$$

is the weight filtration of $\partial M_{g m}\left(\mathrm{~B}^{k}\right)^{e}$ avoiding weights -1 and 0 [W3, Cor. 1.9]. Since $M_{g m}\left(\partial\left(M^{K}\right)^{*}\right) \neq 0$, this shows that both weights $-(k+1)$ and $k$ occur in $\partial M_{g m}\left(\mathrm{~B}^{k}\right)^{e}$.

For $k=0$, we have $\partial M_{g m}\left(\mathrm{~B}^{k}\right)^{e}=\partial M_{g m}\left(M^{K}\right)$. The exact triangle

$$
M_{g m}\left(\partial\left(M^{K}\right)^{*}\right)(1)[1] \longrightarrow \partial M_{g m}\left(M^{K}\right)^{e} \longrightarrow M_{g m}\left(\partial\left(M^{K}\right)^{*}\right) \stackrel{[1]}{\longrightarrow}
$$

is split (cmp. [W3, Ex. 4.12]); therefore both weights -1 and 0 occur in $\partial M_{g m}\left(\mathrm{~B}^{k}\right)^{e}=\partial M_{g m}\left(M^{K}\right)$.

Remark 5.4. (a) The statements on $\partial M_{g m}\left(\mathrm{~B}^{k}\right)^{e}$ from Example 5.3 admit integral versions. More precisely (see [W3, Sect. 3]), they hold in the category $D M_{g m}(\mathbb{Q})$ of geometrical motives over $\mathbb{Q}$, tensored with $\mathbb{Z}[1 /(2 n \cdot k !)]$.

(b) The analysis of the geometry of the boundary of the canonical compactification of $B^{k}$ from $[S$, Sect. 2, 3] can be employed to show directly that the exact triangle

$$
M_{g m}\left(\partial\left(M^{K}\right)^{*}\right)(k+1)[k+1] \longrightarrow \partial M_{g m}\left(\mathrm{~B}^{k}\right)^{e} \longrightarrow M_{g m}\left(\partial\left(M^{K}\right)^{*}\right)[k] \stackrel{[1]}{\longrightarrow}
$$

is induced by an exact triangle

$$
\mathbb{1}_{\partial\left(M^{K}\right)^{*}}[-k] \longrightarrow i^{*} j_{*} \underline{\alpha} \mathcal{V} \longrightarrow \mathbb{1}_{\partial\left(M^{K}\right)^{*}}(-(k+1))[-(k+1)] \stackrel{[1]}{\longrightarrow}
$$

in $D M_{\mathrm{L}, c}\left(\partial\left(M^{K}\right)^{*}\right)$. We leave the details to the reader.

(c) To the author's knowledge, Example 5.3 is the only non-trivial case of a non-compact pure Shimura variety, where the weights in $i^{*} j_{*} \underline{\underline{\alpha}} \mathcal{V}$ can be controlled by purely geometrical, i.e., intrinsically motivic, means.

(d) For arbitrary level $K$, the motive $i^{*} j_{*} \underline{\underline{ }} \mathcal{V} \in D M_{\mathrm{E}, c}\left(\partial\left(M^{K}\right)^{*}\right)$ is still without weights

$$
-(k-1),-(k-2), \ldots, k
$$


and both weights $-k$ and $k+1$ still occur. A motivic proof would run as follows. First, using conjugation in $G L_{2}\left(\mathbb{A}_{f}\right)$, one may assume that $K$ contains the kernel $K_{n}$ modulo $n$, for an integer $n \geq 3$. Then, one analyzes $K / K_{n}$-equivariance of the direct image of the exact triangle

$$
\mathbb{1}_{\partial\left(M^{K_{n}}\right)^{*}}[-k] \longrightarrow i^{*} j_{*} \stackrel{\alpha}{\mathcal{V}} \longrightarrow \mathbb{1}_{\partial\left(M^{K_{n}}\right)^{*}}(-(k+1))[-(k+1)] \stackrel{[1]}{\longrightarrow}
$$

under the finite morphism from $\partial\left(M^{K_{n}}\right)^{*}$ to $\partial\left(M^{K}\right)^{*}$.

Alternatively, use the case $L=\mathbb{Q}$ from Example 5.5 (which relies on realizations).

Example 5.5. Our next example concerns Hilbert-Blumenthal varieties. Fix a totally real number field $L$, and denote by $g$ its degree. The reductive group $G$ equals the fibre product

$$
G:=\mathbb{G}_{m, \mathbb{Q}} \times_{\operatorname{Res}_{L / \mathbb{Q}} \mathbb{G}_{m, L}, \operatorname{det}} \operatorname{Res}_{L / \mathbb{Q}} G L_{2, L}
$$

$\left(\operatorname{Res}_{L / \mathbb{Q}}:=\right.$ the Weil restriction from $L$ to $\left.\mathbb{Q}\right)$. The reflex field equals $\mathbb{Q}$, and $F=F^{\prime}$ is a sub-field of $\mathbb{R}$ containing the images $\sigma(L)$, for $\sigma$ running through the set $I_{L}$ of all real embeddings of $L$. The dominant character $\underline{\alpha}$ is identified with a $(g+1)$-tuple of integers $\left(\left(k_{\sigma}\right)_{\sigma \in I_{L}}, r\right)$, with $k_{\sigma} \geq 0$ and $\bar{r} \equiv \sum_{\sigma \in I_{L}} k_{\sigma}$ mod 2: note that

$$
\left(\operatorname{Res}_{L / \mathbb{Q}} \mathrm{GL}_{2, L}\right) \times_{\mathbb{Q}} F \stackrel{\sim}{\longrightarrow} \prod_{\sigma \in I_{L}} \mathrm{GL}_{2, F},
$$

and under that isomorphism,

$$
G \times_{\mathbb{Q}} F \stackrel{\sim}{\longrightarrow}\left\{\left(M_{\sigma}\right)_{\sigma \in I_{L}} \in \prod_{\sigma \in I_{L}} \mathrm{GL}_{2, F}, \operatorname{det}\left(M_{\sigma}\right)=\operatorname{det}\left(M_{\eta}\right) \quad \forall \sigma, \eta \in I_{L}\right\} .
$$

Choosing $T \subset G \times_{\mathbb{Q}} F \subset \prod_{\sigma \in I_{L}} G_{2, F}$ to be equal to the sub-group of elements having diagonal entries at each $\sigma \in I_{L}$, we have

$$
\underline{\alpha}: T \longrightarrow \mathbb{G}_{m, F},\left(\operatorname{diag}\left(a_{\sigma}, a_{\sigma}^{-1} q\right)\right)_{\sigma \in I_{L}} \longmapsto\left(\prod_{\sigma \in I_{L}} a_{\sigma}^{k_{\sigma}}\right) \cdot q^{-\frac{r+\sum_{\sigma} k_{\sigma}}{2}} .
$$

The canonical representation $V$ equals the (2g-dimensional) Weil restriction of the standard two-dimensional representation of $G L_{2, L}$. Thus,

$$
V \times_{\mathbb{Q}} F \stackrel{\sim}{\longrightarrow} \bigoplus_{\sigma \in I_{L}} V_{\sigma}
$$

where for $\sigma \in I_{L}$, we denote by $V_{\sigma}$ the standard two-dimensional representation of $G L_{2, F}$, seen as the $\sigma$-component of $\left(\operatorname{Res}_{L / \mathbb{Q}} \mathrm{GL}_{2, L}\right) \times_{\mathbb{Q}} F$. It is the image of an idempotent endomorphism $e_{\sigma}$ of $V \times_{\mathbb{Q}} F \in \operatorname{Rep}\left(G_{F}\right)$, whose kernel equals $\oplus_{\eta \neq \sigma} V_{\eta}$. Then, setting $k:=\sum_{\sigma} k_{\sigma}$,

$$
V_{\underline{\alpha}}=\left(\bigotimes_{\sigma \in I_{L}} \operatorname{Sym}^{k_{\sigma}} V_{\sigma}\right)\left(-\frac{r+k}{2}\right) \in \operatorname{Rep}\left(G_{F}\right) \text {. }
$$


Theorem 5.1 therefore shows that

$$
\underline{\alpha} \mathcal{V}=\left(\bigotimes_{\sigma \in I_{L}} \operatorname{Sym}^{k_{\sigma}} \pi_{*}^{1} \mathbb{1}_{\mathrm{B}}^{e_{\sigma}}\right)\left(\frac{-r+k}{2}\right)[-r+k] \in C H M\left(M^{K}\right)_{F},
$$

where $\pi: \mathrm{B} \rightarrow M^{K}$ is the universal Abelian $g$-fold, and for every $\sigma \in I_{L}$, we denote by $e_{\sigma}: \pi_{*}^{1} \mathbb{1}_{\mathrm{B}} \rightarrow \pi_{*}^{1} \mathbb{1}_{\mathrm{B}}$ the idempotent endomorphism of $\pi_{*}^{1} \mathbb{1}_{\mathrm{B}}$ induced by functoriality.

We claim that for any (neat) level $K$, the following is true: the motive $i^{*} j_{*} \underline{\underline{V}}$ is zero unless $\underline{\alpha}$ is parallel, i.e., unless all $k_{\sigma}$ are equal to each other. Furthermore, $i^{*} j_{*} \underline{\alpha} \mathcal{V}$ is without weights

$$
-(k-1),-(k-2), \ldots, k,
$$

and both weights $-k$ and $k+1 d o$ occur in $i^{*} j_{*} \underline{\alpha} \mathcal{V}$, provided $\underline{\alpha}$ is parallel. In particular, $\underline{\alpha} \mathcal{V}$ belongs to $C H M\left(M^{K}\right)_{F, \partial w \neq 0,1} \subset C H M\left(M^{K}\right)_{F}$ if and only if $k=\sum_{\sigma} k_{\sigma} \geq 1$, i.e., if and only if at least one of the tensor components $\operatorname{Sym}^{k_{\sigma}} V_{\sigma}$ of $V_{\underline{\alpha}}$ is regular.

In order to show the claim, note first that as in Example 5.3, we may assume that $r=k$, i.e., that

$$
\underline{\alpha} \mathcal{V}=\bigotimes_{\sigma \in I_{L}} \operatorname{Sym}^{k_{\sigma}} \pi_{*}^{1} \mathbb{1}_{\mathrm{B}}^{e_{\sigma}}
$$

The Chow motive $\underline{\underline{V}}$ thus equals a direct factor of $\pi_{k, *} \mathbb{1}_{\mathrm{B}^{k}}$, where we denote by $\pi_{k}$ the projection of the $k$-fold fibre product $\mathrm{B}^{k}$ to $M^{K}$. For a concrete description of the associated idempotent endomorphism

$$
e \in \mathrm{CH}_{g(k+1)}\left(\mathrm{B}^{k} \times_{M^{K}} \mathrm{~B}^{k}\right) \otimes_{\mathbb{Z}} F,
$$

we refer to [W4, Lemma 3.4].

Next, by $\left[\mathrm{W} 4\right.$, Thm. 3.5, 3.6], the motive $\partial M_{g m}\left(\mathrm{~B}^{k}\right)^{e} \in D M_{\mathrm{b}, c}(\mathbb{Q})_{F}$ is zero if $\underline{\alpha}$ is not parallel, and it is without weights $-k,-(k-1), \ldots, k-1$. Now as in Example 5.3, the scheme of cusps $\partial\left(M^{K}\right)^{*}$ is finite over $\operatorname{Spec} \mathbb{Q}$. According to Corollary [3.16, the motive $i^{*} j_{*} \underline{\alpha} \mathcal{V}$ is therefore zero unless $\underline{\alpha}$ is parallel, and it is without weights $-(k-1),-(k-2), \ldots, k$.

It remains to show that if $\underline{\alpha}$ is parallel, then both weights $-k$ and $k+$ 1 occur in $i^{*} j_{*} \underline{\mathcal{Q}} \mathcal{V}$, or equivalently, both weights $-(k+1)$ and $k$ occur in $\partial M_{g m}\left(\mathrm{~B}^{k}\right)^{e}$. All $k_{\sigma}$ are equal to each other, say

$$
k_{\sigma}=s \geq 0 \quad \forall \sigma \in I_{L} .
$$

Thus, $k=g \cdot s$, and

$$
V_{\underline{\alpha}}=\left(\bigotimes_{\sigma \in I_{L}} \operatorname{Sym}^{s} V_{\sigma}\right)(-k),
$$

which is isomorphic to $\bigotimes_{\sigma \in I_{L}} \operatorname{Sym}^{s} V_{\sigma}^{\vee}$ (where we denote by $V_{\sigma}^{\vee}$ the dual of $V_{\sigma}$, for all $\left.\sigma \in I_{L}\right)$. 
By [W4, Prop. 2.5], the motive $\partial M_{g m}\left(\mathrm{~B}^{k}\right)^{e}$ realizes to give boundary cohomology

$$
\left(\left(\partial H^{n}\left(\mathrm{~B}^{k}(\mathbb{C}), \mathbb{Q}\right) \otimes_{\mathbb{Q}} F\right)^{e}\right)_{n \in \mathbb{Z}} .
$$

By [W4, Prop. 4.5], for all $n \in \mathbb{Z}$, there are isomorphisms of Hodge structures

$$
\left(\partial H^{n}\left(\mathrm{~B}^{k}(\mathbb{C}), \mathbb{Q}\right) \otimes_{\mathbb{Q}} F\right)^{e} \stackrel{\sim}{\longrightarrow} \partial H^{n-k}\left(M^{K}(\mathbb{C}), \mu_{\mathbf{H}}\left(V_{\underline{\alpha}}\right)\right)
$$

(note that the weight of $V_{\underline{\alpha}}$ equals $k$ ). According to the proof of [W4,

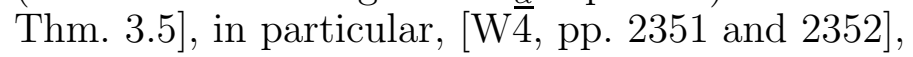

$$
\partial H^{0}\left(M^{K}(\mathbb{C}), \mu_{\mathbf{H}}\left(V_{\underline{\alpha}}\right)\right)
$$

is non-zero, and pure of weight 0 , while

$$
\partial H^{2 g-1}\left(M^{K}(\mathbb{C}), \mu_{\mathbf{H}}\left(V_{\underline{\alpha}}\right)\right)
$$

is non-zero, and pure of weight $2(k+g)$. Therefore,

$$
\left(\partial H^{k}\left(\mathrm{~B}^{k}(\mathbb{C}), \mathbb{Q}\right) \otimes_{\mathbb{Q}} F\right)^{e} \neq 0
$$

is pure of weight 0 , and

$$
\left(\partial H^{k+2 g-1}\left(\mathrm{~B}^{k}(\mathbb{C}), \mathbb{Q}\right) \otimes_{\mathbb{Q}} F\right)^{e} \neq 0
$$

is pure of weight $2(k+g)$. Therefore, weights $0-k=-k$ and $2(k+g)-$ $(k+2 g-1)=k+1$ occur in the realization of $\partial M_{g m}\left(\mathrm{~B}^{k}\right)^{e}$. Given that the realization on geometrical motives is contravariant, and exchanges the signs of weights, this implies in particular that weights $-(k+1)$ and $k$ occur in $\partial M_{g m}\left(\mathrm{~B}^{k}\right)^{e}$.

Remark 5.6. The proofs of $[\mathrm{W} 4$, Thm. 3.5, 3.6] rely on the fact that $\partial M_{g m}\left(\mathrm{~B}^{k}\right)$ is a Dirichlet-Tate motive over $\mathbb{Q}$, and that on such motives, the realizations are weight conservative [W7, Cor. 3.10 (c)].

Example 5.7. The third example concerns Picard varieties. Fix a $C M$ field $L$, and denote by $2 g$ its degree. Fix a three-dimensional $L$-vector space $V^{\prime}$, together with an $L$-valued non-degenerate Hermitian form $J$, such that for every $\sigma$ in the set $I_{L}$ of complex embeddings of $L$, the form $J_{\sigma}:=J \otimes_{L, \sigma} \mathbb{C}$ is of signature $(2,1)$. Fix a $C M$-type $\Psi$ of $L$; thus, the set $I_{L}$ is the disjoint union of $\Psi$ and of its conjugate. The reductive group $G$ equals the group of unitary similitudes

$$
G:=G U\left(V^{\prime}, J\right) \subset \operatorname{Res}_{L / \mathbb{Q}} \operatorname{GL}_{L}\left(V^{\prime}\right) .
$$

Thus, for any $\mathbb{Q}$-algebra $R$, the group $G(R)$ equals

$$
\left\{g \in \mathrm{GL}_{L \otimes_{\mathbb{Q}} R}\left(V^{\prime} \otimes_{\mathbb{Q}} R\right), \exists \lambda(g) \in R^{*}, J(g \bullet, g \bullet)=\lambda(g) \cdot J(\bullet, \bullet)\right\} .
$$

In particular, the similitude norm $\lambda(g)$ defines a canonical morphism

$$
\lambda: G \longrightarrow \mathbb{G}_{m, \mathbb{Q}} \text {. }
$$


Then $F=F^{\prime}$ is a sub-field of $\mathbb{C}$ containing the images $\sigma(L)$, for all $\sigma \in I_{L}$. There is an isomorphism

$$
G \times_{\mathbb{Q}} F \stackrel{\sim}{\longrightarrow}\left(\prod_{\sigma \in \Psi} \mathrm{GL}_{F}\left(V_{F}^{\prime}\right)\right) \times_{F} \mathbb{G}_{m, F}
$$

([Cl, p. 4]; see [W8, p. 364] for the case $g=1$ ).

Fix a basis of $V^{\prime}$; thus,

$$
G \times_{\mathbb{Q}} F \stackrel{\sim}{\longrightarrow}\left(\prod_{\sigma \in \Psi} \mathrm{GL}_{3, F}\right) \times_{F} \mathbb{G}_{m, F}
$$

The dominant character $\underline{\alpha}$ is identified with a $(3 g+1)$-tuple of integers $\left(\left(k_{1, \sigma}, k_{2, \sigma}, c_{\sigma}\right)_{\sigma \in \Psi}, r\right)$, with $k_{1, \sigma} \geq k_{2, \sigma} \geq 0$,

$$
c_{\sigma} \equiv k_{1, \sigma}+k_{2, \sigma} \quad \bmod 2, \quad \text { and } \quad r \equiv \sum_{\sigma \in \Psi} \frac{c_{\sigma}+k_{1, \sigma}+k_{2, \sigma}}{2} \bmod 2:
$$

choosing $T \subset\left(\prod_{\sigma \in \Psi} \mathrm{GL}_{3, F}\right) \times_{F} \mathbb{G}_{m, F}$ to be equal to the sub-group of elements having diagonal entries at each $\sigma \in \Psi$, the character $\underline{\alpha}$ sends

$$
\left(\left(\operatorname{diag}\left(a_{\sigma}, a_{\sigma}^{-1} b_{\sigma}, b_{\sigma}^{-1} q_{\sigma}\right)\right)_{\sigma \in \Psi}, f\right) \in T(F)
$$

to

$$
\left(\prod_{\sigma \in \Psi} a_{\sigma}^{k_{1, \sigma}-k_{2, \sigma}} b_{\sigma}^{k_{2, \sigma}} q_{\sigma}^{\frac{c_{\sigma}-\left(k_{1, \sigma}+k_{2, \sigma}\right)}{2}}\right) \cdot f^{-\frac{1}{2}\left(r+\sum_{\sigma} \frac{3 c_{\sigma}-\left(k_{1, \sigma}+k_{2, \sigma}\right)}{2}\right)} \in \mathbb{G}_{m}(F) .
$$

Under this normalization, the similitude norm $\lambda$ corresponds to the character $\underline{\alpha}=\left((0,0,0)_{\sigma \in \Psi},-2\right)$. The corresponding irreducible representation $V_{\lambda}$ of $\bar{G}_{F}$, and actually, of $G$, is the Tate twist $\mathbb{Q}(1)$. It follows from Theorem 5.1 (c) that as far as control of weights is concerned, we may replace a given $\underline{\alpha}=$ $\left(\left(k_{1, \sigma}, k_{2, \sigma}, c_{\sigma}\right)_{\sigma \in \Psi}, r\right)$ by $\left(\left(k_{1, \sigma}, k_{2, \sigma}, c_{\sigma}\right)_{\sigma \in \Psi}, r^{\prime}\right)$, with $r^{\prime} \equiv r \bmod 2$.

We claim that the motive $\underline{\alpha} \mathcal{V}$ belongs to $C H M\left(M^{K}\right)_{F, \partial w \neq 0,1}$ if $\underline{\alpha}$ is regular, i.e., if for all $\sigma \in \Psi$, we have

$$
k_{1, \sigma}>k_{2, \sigma}>0 .
$$

In order to show the claim, it will be useful to compare our parametrization of characters to that of [Cl, Sect. 3]. There, the standard basis of characters of the split torus $T$ is used. First, write $\left(\left(a_{\sigma}, b_{\sigma}, \gamma_{\sigma}\right)_{\sigma \in \Psi}, d\right)$ instead of $\left(\left(a_{\sigma}, b_{\sigma}, c_{\sigma}\right)_{\sigma \in \Psi}, d\right)$ as in [loc. cit.], and $\lambda\left(\left(a_{\sigma}, b_{\sigma}, \gamma_{\sigma}\right)_{\sigma \in \Psi}, d\right)$ for the associated character. We then leave it to the reader to verify that the change of parameters $\lambda\left(\left(a_{\sigma}, b_{\sigma}, \gamma_{\sigma}\right)_{\sigma \in \Psi}, d\right) \leftrightarrow\left(\left(k_{1, \sigma}, k_{2, \sigma}, c_{\sigma}\right)_{\sigma \in \Psi}, r\right)$ is the following: the character $\left(\left(k_{1, \sigma}, k_{2, \sigma}, c_{\sigma}\right)_{\sigma \in \Psi}, r\right)$ of $T$ equals

$$
\lambda\left(\left(\frac{c_{\sigma}+k_{1, \sigma}-k_{2, \sigma}}{2}, \frac{c_{\sigma}-k_{1, \sigma}+k_{2, \sigma}}{2}, \frac{c_{\sigma}-\left(k_{1, \sigma}+k_{2, \sigma}\right)}{2}\right)_{\sigma \in \Psi}, d\right),
$$


with $d=-\frac{1}{2}\left(r+\sum_{\sigma} \frac{3 c_{\sigma}-\left(k_{1, \sigma}+k_{2, \sigma}\right)}{2}\right)$. The character $\lambda\left(\left(a_{\sigma}, b_{\sigma}, \gamma_{\sigma}\right)_{\sigma \in \Psi}, d\right)$ equals

$$
\left(\left(a_{\sigma}-\gamma_{\sigma}, b_{\sigma}-\gamma_{\sigma}, a_{\sigma}+b_{\sigma}\right)_{\sigma \in \Psi},-\left(2 d+\sum_{\sigma}\left(a_{\sigma}+b_{\sigma}+\gamma_{\sigma}\right)\right)\right) \text {. }
$$

The character $\lambda\left(\left(a_{\sigma}, b_{\sigma}, \gamma_{\sigma}\right)_{\sigma \in \Psi}, d\right)$ is dominant if and only if for for all $\sigma \in \Psi$,

$$
a_{\sigma} \geq b_{\sigma} \geq \gamma_{\sigma}
$$

and it is regular if and only if for for all $\sigma \in \Psi$,

$$
a_{\sigma}>b_{\sigma}>\gamma_{\sigma} \text {. }
$$

To replace $\underline{\alpha}=\left(\left(k_{1, \sigma}, k_{2, \sigma}, c_{\sigma}\right)_{\sigma \in \Psi}, r\right)$ by $\left(\left(k_{1, \sigma}, k_{2, \sigma}, c_{\sigma}\right)_{\sigma \in \Psi}, r^{\prime}\right)$, with $r^{\prime} \equiv r$ $\bmod 2$, means to replace $\lambda\left(\left(a_{\sigma}, b_{\sigma}, \gamma_{\sigma}\right)_{\sigma \in \Psi}, d\right)$ by $\lambda\left(\left(a_{\sigma}, b_{\sigma}, \gamma_{\sigma}\right)_{\sigma \in \Psi}, d^{\prime}\right)$. This together with [Cl, Prop.3.2] shows that as far as our weight estimates are concerned, we may assume, by modifying the value of $r$ if necessary, that the Chow motive $\underline{\alpha} \mathcal{V}$ equals a direct factor of $\pi_{r, *} \mathbb{1}_{\mathrm{B}^{r}}$, where we denote by $\pi_{r}$ the projection of the $r$-fold fibre product $\mathrm{B}^{r}$ to $M^{K}$. Let $e$ be the associated idempotent endomorphism of $\pi_{r, *} \mathbb{1}_{\mathrm{B}^{r}}$.

By [Cl, Thm. 3.6], the motive $\partial M_{g m}\left(\mathrm{~B}^{r}\right)^{e} \in D M_{\mathrm{L}, c}(\operatorname{Spec} E)_{F}$ is without weights -1 and 0 if $\alpha$ is regular. Now as in Examples 5.3 and 5.5, the scheme of cusps $\partial\left(M^{\bar{K}}\right)^{*}$ is finite over Spec $E$. According to Corollary 3.16, the motive $\underline{\alpha} \mathcal{V}$ therefore lies in $C H M\left(M^{K}\right)_{F, \partial w \neq 0,1}$ if $\underline{\alpha}$ is regular.

Remark 5.8. (a) For $g=1$, it is shown in [W8, Thm. 3.8] that regularity of $\underline{\alpha}$ is not only sufficient, but also necessary for $\underline{\alpha} \mathcal{V}$ to belong to $C H M\left(M^{\bar{K}}\right)_{F, \partial w \neq 0,1}$. In fact, a precise interval of weights avoided by $i^{*} j_{*} \underline{\alpha} \mathcal{V}$ is given: putting $k:=\min \left(k_{1, \sigma}-k_{2, \sigma}, k_{2, \sigma}\right)$ (for the unique $\sigma$ in $\Psi$ ), the motive $i^{*} j_{*} \underline{\underline{V}}$ is without weights

$$
-(k-1),-(k-2), \ldots, k,
$$

and both weights $-k$ and $k+1$ do occur in $i^{*} j_{*} \underline{\alpha} \mathcal{V}$ ([W8, Thm. 3.8], Corollary 3.16).

(b) As soon as $g \geq 2$, regularity of $\alpha$ is no longer necessary for $\underline{\alpha} \mathcal{V}$ to belong to CHM( $\left.M^{K}\right)_{F, \partial w \neq 0,1}$, as illustrated by [Cl, Prop. 3.8]. By checking the details of the computations leading to the proof of [Cl, Thm. 3.6] (see in particular $\left[\mathrm{Cl}\right.$, pp. 21-24]), one sees that the motive $\underline{\alpha} \mathcal{V}$ belongs to $C H M\left(M^{K}\right)_{F, \partial w \neq 0,1}$ if and only if at least one of the components $\left(k_{1, \sigma}, k_{2, \sigma}, c_{\sigma}\right)$ of $\underline{\alpha}$ is regular, i.e., if and only if

$$
k_{1, \sigma}>k_{2, \sigma}>0
$$

for some $\sigma \in \Psi$. Note that this condition is equivalent to saying that $\underline{\alpha}$ is not Kostant-parallel for $m=-1$ in the terminology of [Cl, Def. 3.2].

It is proved [Cl, Prop. 3.7] that $i^{*} j_{*} \underline{\underline{\alpha}} \mathcal{V} \neq 0$ if and only if $\underline{\alpha}$ is Kostantparallel for some integer $m \geq-1$ (this is the case in particular if $\underline{\alpha}$ is parallel, i.e., if all pairs $\left(k_{1, \sigma}, k_{2, \sigma}\right)$ are equal to each other). Thus, assume $i^{*} j_{*} \underline{\mathcal{V}} \neq 0$, and denote by $m$ the minimal value for which $\underline{\alpha}$ is Kostant-parallel. Define 
$n$ as the number of $\sigma \in \Psi$ for which $k_{1, \sigma}>m$. A close analysis of [Cl, pp. 21-24] reveals that $i^{*} j_{*} \underline{\alpha} \mathcal{V}$ is without weights

$$
-(m g+n-1),-(m g+n-2), \ldots, m g+n,
$$

and that both weights $-(m g+n)$ and $m g+n+1$ do occur in $i^{*} j_{*} \underline{\alpha}$.

(c) The proofs of [W8, Thm. 3.8] and [Cl, Thm. 3.6] rely on the fact that $\partial M_{g m}\left(\mathrm{~B}^{r}\right)$ is a motive of Abelian type over $E$, and that on such motives, the realizations are weight conservative [W8, Thm. 1.13].

Remark 5.9. None of the examples treated so far necessitates the use of the new criteria on absence of weights in the boundary proved in Section 4. Indeed, weights were controlled, respectively, by purely geometrical means (Example 5.3), by using weight conservativity of the restriction of the realizations to Dirichlet-Tate motives (Example 5.5), and by using weight conservativity of the restriction of the realizations to motives of Abelian type over a point (Example 5.7).

All of them could be proved using Corollary 4.6, since the main assumption: $i^{*} j_{*} \underline{\alpha} \mathcal{V}$ of Abelian type, is satisfied.

Example 5.10. Our last example concerns Hilbert-Siegel varieties. Fix a totally real number field $L$, and denote by $g$ its degree. Fix a four-dimensional $L$-vector space $V^{\prime}$, together with an $L$-valued non-degenerate symplectic bilinear form $J$. The reductive group $G$ equals the group of symplectic similitudes

$$
G:=G S p\left(V^{\prime}, J\right) \subset \operatorname{Res}_{L / \mathbb{Q}} \operatorname{GL}_{L}\left(V^{\prime}\right) .
$$

Thus, for any $\mathbb{Q}$-algebra $R$, the group $G(R)$ equals

$$
\left\{g \in \mathrm{GL}_{L \otimes_{\mathbb{Q}} R}\left(V^{\prime} \otimes_{\mathbb{Q}} R\right), \exists \lambda(g) \in R^{*}, J(g \bullet, g \bullet)=\lambda(g) \cdot J(\bullet, \bullet)\right\} .
$$

In particular, the similitude norm $\lambda(g)$ defines a canonical morphism

$$
\lambda: G \longrightarrow \mathbb{G}_{m, \mathbb{Q}} \text {. }
$$

The reflex field equals $\mathbb{Q}$, and $F=F^{\prime}$ is a sub-field of $\mathbb{R}$ containing the images $\sigma(L)$, for $\sigma$ running through the set $I_{L}$ of all real embeddings of $L$.

Fix a symplectic basis of $V$, in which $J$ acquires the $4 \times 4$-matrix

$$
\left(\begin{array}{cc}
0 & I_{2} \\
-I_{2} & 0
\end{array}\right)
$$

thus,

$$
G \times \times_{\mathbb{Q}} F \longleftrightarrow \operatorname{Res}_{L / \mathbb{Q}} G S p_{4, L},
$$

where $G S p_{4, L}$ denotes the sub-group of $G L_{4, \mathbb{Q}}$ of matrices $g$ satisfying the relation

$$
{ }^{t} g J g=\lambda(g) \cdot J .
$$


More precisely,

$$
G:=\mathbb{G}_{m, \mathbb{Q}} \times_{\operatorname{Res}_{L / \mathbb{Q}} \mathbb{G}_{m, L}, \operatorname{det}} \operatorname{Res}_{L / \mathbb{Q}} G S p_{4, L} .
$$

The dominant character $\underline{\alpha}$ is identified with a $(2 g+1)$-tuple of integers $\left(\left(k_{1, \sigma}, k_{2, \sigma}\right)_{\sigma \in I_{L}}, r\right)$, with $k_{1, \sigma} \geq k_{2, \sigma} \geq 0$ and $r \equiv \sum_{\sigma \in I_{L}}\left(k_{1, \sigma}+k_{2, \sigma}\right) \bmod 2$ : choosing $T \subset G \times_{\mathbb{Q}} F \subset \prod_{\sigma \in I_{L}} G S p_{4, F}$ to be equal to the sub-group of elements having diagonal entries at each $\sigma \in I_{L}$, the character $\underline{\alpha}$ sends

$$
\left(\operatorname{diag}\left(a_{\sigma}, b_{\sigma}, a_{\sigma}^{-1} q, b_{\sigma}^{-1} q\right)\right)_{\sigma \in I_{L}} \in T(F)
$$

to

$$
\left(\prod_{\sigma \in I_{L}} a_{\sigma}^{k_{1, \sigma}}\right) \cdot\left(\prod_{\sigma \in I_{L}} b_{\sigma}^{k_{2, \sigma}}\right) \cdot q^{-\frac{r+\sum_{\sigma}\left(k_{1, \sigma}+k_{2, \sigma}\right)}{2}} .
$$

Using the criterion from Corollary 4.6 (b), it is shown in [Ca, Cor. 2.1.0.4] that the motive $\underline{\alpha} \mathcal{V}$ belongs to $C H M\left(M^{K}\right)_{F, \partial w \neq 0,1}$ if at least one of the components $\left(k_{1, \sigma}, k_{2, \sigma}\right)$ of $\underline{\alpha}$ is regular, i.e., if

$$
k_{1, \sigma}>k_{2, \sigma}>0
$$

for some $\sigma \in I_{L}$.

Remark 5.11. (a) For $g=1$, it is shown in [W11, Thm. 1.6] that regularity of $\underline{\alpha}$ is not only sufficient, but also necessary for $\underline{\alpha} \mathcal{V}$ to belong to $C H M\left(M^{\underline{K}}\right)_{F, \partial w \neq 0,1}$. In fact, a precise interval of weights avoided by $i^{*} j_{*} \underline{\alpha} \mathcal{V}$ is given: putting $k:=\min \left(k_{1, \sigma}-k_{2, \sigma}, k_{2, \sigma}\right)$ (for the unique $\sigma$ in $I_{L}$ ), the motive $i^{*} j_{*} \underline{\underline{V}} \mathcal{V}$ is without weights

$$
-(k-1),-(k-2), \ldots, k,
$$

and both weights $-k$ and $k+1$ do occur in $i^{*} j_{*} \underline{\underline{\alpha}} \mathcal{V}$.

(b) As soon as $g \geq 2$, regularity of one of the components of $\underline{\alpha}$ is no longer necessary for $\underline{\alpha} \mathcal{V}$ to belong to $C H M\left(M^{K}\right)_{F, \partial w \neq 0,1}$. Indeed, according to Ca, Cor. 2.1.0.4], for the motive $\underline{\alpha} \mathcal{V}$ not to belong to $C H M\left(M^{K}\right)_{F, \partial w \neq 0,1}$, it is necessary and sufficient that none of the components $\left(k_{1, \sigma}, k_{2, \sigma}\right)$ of $\underline{\alpha}$ is regular, and that in addition all $k_{2, \sigma}$ are equal to each other.

For $g=2$ one might thus choose $\underline{\alpha}$ equal to $((1,0),(1,1), 3)$, to obtain an example of a character none of whose components is regular, but whose associated motive $\underline{\alpha} \mathcal{V}$ belongs nonetheless to $C H M\left(M^{K}\right)_{F, \partial w \neq 0,1}$.

(c) In [Ca, Thm. 2.1.0.3], an interval of weights avoided by $i^{*} j_{*} \underline{\underline{\alpha}} \mathcal{V}$, sharp in most cases, is given for any value of $g$. As in the case of Picard varieties, the notion of Kostant-parallelism occurs. But in addition, the notion of corank is needed. We refer to [loc. cit.] for the definition of the corank, and for the precise formulae.

(d) As was the case in our earlier examples, the above weight estimates are valid for any neat level $K$. 
Remark 5.12. According to Corollary 4.6 (c) and Proposition 3.8, the weight estimates for $i^{*} j_{*} \underline{\alpha} \mathcal{V}$ induce weight estimates for the corresponding $e$-part $\partial M_{g m}\left(\mathrm{~B}^{r}\right)^{e}$ of the boundary motive $\partial M_{g m}\left(\mathrm{~B}^{r}\right)$.

Contrary to the cases treated in Examples 5.3, 5.5 and 5.7, HilbertSiegel varieties have a boundary $\partial\left(M^{K}\right)^{*}$ of strictly positive dimension (equal to the degree $g$ of the totally real number field $L$ ). More seriously, that boundary is not of Abelian type over $\operatorname{Spec} \mathbb{Q}$. Thus, our earlier results on weight conservativity [W7, W8 cannot be employed to control the weights in $\partial\left(M^{K}\right)^{*}$ directly from those in the boundary cohomology of $M^{K}$.

The strategy set out in Section 4 , i.e., the detour via relative motives over $\left(M^{K}\right)^{*}$, is therefore needed in order to treat Example 5.10.

Given our examples, the following seems to be justified.

Question 5.13 ([W12, Conj. A]). Let $M^{K}$ be a Shimura variety of $P E L$ type, associated to pure Shimura data $(G, \mathcal{H})$ and a neat level $K$. Denote by $j$ the open immersion of $M^{K}$ into its Baily-Borel compactification $\left(M^{K}\right)^{*}$, and by $i$ the closed immersion of the boundary $\partial\left(M^{K}\right)^{*}$ of $\left(M^{K}\right)^{*}$. Let $V_{\underline{\alpha}}$ be an irreducible regular representation of $G$.

Does the motive $\underline{\alpha} \mathcal{V}=\widetilde{\mu}\left(V_{\underline{\alpha}}\right)$ belong to $C H M\left(M^{K}\right)_{F, \partial w \neq 0,1}$ ?

Our examples actually suggest that weaker conditions on $\underline{\alpha}$ are still sufficient for $\underline{\alpha} \mathcal{V}$ to belong to $C H M\left(M^{K}\right)_{F, \partial w \neq 0,1}$. We refer to [W12] for stronger versions of Question 5.13,

\section{References}

[Anc] G. Ancona, Décomposition de motifs abéliens, manuscripta math. 146 (2015), 307-328.

[And] Y. André, Une introduction aux motifs, Panoramas et Synthèses 17, Soc. Math. France (2004).

[AK] Y. André, B. Kahn, Nilpotence, radicaux et structures monoïdales, avec un appendice de P. O'Sullivan, Rend. Sem. Mat. Univ. Padova 108 (2002), 107-291, Erratum, 113 (2005), 125-128.

[Ay] J. Ayoub, Note sur les opérations de Grothendieck et la réalisation de Betti, J. Inst. Math. Jussieu 9 (2010), 225-263.

[BBD] A.A. Beilinson, J. Bernstein, P. Deligne, Faisceaux pervers, in B. Teissier, J.L. Verdier (eds.), Analyse et topologie sur les espaces singuliers (I), Astérisque 100, Soc. Math. France (1982). 
[B1] M.V. Bondarko, Weight structures vs. t-structures; weight filtrations, spectral sequences, and complexes (for motives and in general), J. KTheory 6 (2010), 387-504.

[B2] M.V. Bondarko, $\mathbb{Z}[1 / p]$-motivic resolution of singularities, Compositio Math. 147 (2011), 1434-1446.

[B3] M.V. Bondarko, Mixed motivic sheaves (and weights for them) exist if 'ordinary' mixed motives do, Compositio Math. 151 (2015), 917-956.

[Ca] M. Cavicchi, On the boundary and intersection motives of genus 2 Hilbert-Siegel varieties, preprint, May 2018, 39 pages, available on arXiv.org under https://arxiv.org/abs/1805.00440

[CD1] D.-C. Cisinski, F. Déglise, Local and stable homological algebra in Grothendieck abelian categories, Homology, Homotopy and Applications 11 (2009), 219-260.

[CD2] D.-C. Cisinski, F. Déglise, Triangulated categories of mixed motives, preprint, December 2009, version dated December 18, 2012, 279 pages, available on arXiv.org under https://arxiv.org/abs/0912.2110

[CD3] D.-C. Cisinski, F. Déglise, Etale motives, Compositio Math. 152 (2016), 556-666.

[CM] M.A.A. de Cataldo, L. Migliorini, The Chow motive of semismall resolutions, Math. Res. Lett. 11 (2004), 151-170.

[Cl] G. Cloître, On the interior motive of certain Shimura varieties: the case of Picard varieties, preprint, May 2017, 32 pages, available on arXiv.org under https://arxiv.org/abs/1705.03235

[D] F. Déglise, Around the Gysin triangle II, Doc. Math. 13 (2008), 613-675.

[DM] C. Deninger, J.P. Murre, Motivic decomposition of abelian schemes and the Fourier transform, J. reine angew. Math 422 (1991), 201-219.

[E] T. Ekedahl, On the adic formalism, in P. Cartier et al. (eds.), The Grothendieck Festschrift, Volume II, Prog. in Math. 87, BirkhäuserVerlag (1990), 197-218.

[F] J. Fangzhou, Borel-Moore motivic homology and weight structure on mixed motives, Math. Z. 283 (2016), 1149-1183.

[FV] E.M. Friedlander, V. Voevodsky, Bivariant cycle cohomology, Chapter 4 of V. Voevodsky, A. Suslin, E.M. Friedlander, Cycles, Transfers, and Motivic Homology Theories, Ann. of Math. Studies 143, Princeton Univ. Press (2000). 
[H] D. Hébert, Structures de poids à la Bondarko sur les motifs de Beilinson, Compositio Math. 147 (2011), 1447-1462.

[K] S. Kelly, Voevodsky motives and $\ell$ dh-descent, Astérisque 391, Soc. Math. France (2017).

[L] M. Levine, Smooth Motives, in R. de Jeu, J.D. Lewis (eds.), Motives and Algebraic Cycles. A Celebration in Honour of Spencer J. Bloch, Fields Institute Communications 56, Amer. Math. Soc. (2009), 175-231.

[S] A.J. Scholl, Motives for modular forms, Invent. Math. 100 (1990), 419430.

[Va] V. Vaish, Motivic intersection complex of certain Shimura varieties, preprint, January 2018, 17 pages, available on arXiv.org under https://arxiv.org/abs/1801.09243

[Vo] V. Voevodsky, Triangulated categories of motives over a field, Chapter 5 of V. Voevodsky, A. Suslin, E.M. Friedlander, Cycles, Transfers, and Motivic Homology Theories, Ann. of Math. Studies 143, Princeton Univ. Press (2000).

[W1] J. Wildeshaus, The canonical construction of mixed sheaves on mixed Shimura varieties, in: Realizations of Polylogarithms, Lect. Notes Math. 1650, Springer-Verlag (1997), 77-140.

[W2] J. Wildeshaus, The boundary motive: definition and basic properties, Compositio Math. 142 (2006), 631-656.

[W3] J. Wildeshaus, Chow motives without projectivity, Compositio Math. 145 (2009), 1196-1226.

[W4] J. Wildeshaus, On the Interior Motive of Certain Shimura Varieties: the Case of Hilbert-Blumenthal varieties, Int. Math. Res. Notices 2012 (2012), 2321-2355.

[W5] J. Wildeshaus, Motivic intersection complex, in J.I. Burgos and J. Lewis (eds.), Regulators III. Proceedings of the conference held at the University of Barcelona, July 11-23, 2010, Contemp. Math. 571, Amer. Math. Soc. (2012), 255-276.

[W6] J. Wildeshaus, Boundary motive, relative motives and extensions of motives, in J.-B. Bost and J.-M. Fontaine (eds.), Autour des motifs Ecole d'été Franco-Asiatique de Géométrie Algébrique et de Théorie des Nombres. Vol. II, Panoramas et Synthèses 41, Soc. Math. France (2013), 143-185. 
[W7] J. Wildeshaus, Notes on Artin-Tate motives, in J.-B. Bost and J.M. Fontaine (eds.), Autour des motifs - Ecole d'été Franco-Asiatique de Géométrie Algébrique et de Théorie des Nombres. Vol. III, Panoramas et Synthèses 49, Soc. Math. France (2016), 101-131.

[W8] J. Wildeshaus, On the interior motive of certain Shimura varieties: the case of Picard surfaces, manuscripta math. 148 (2015), 351-377.

[W9] J. Wildeshaus, Intermediate extension of Chow motives of Abelian type, Adv. Math. 305 (2017), 515-600.

[W10] J. Wildeshaus, Weights and conservativity, Algebraic Geometry 5 (2018), 686-702.

[W11] J. Wildeshaus, On the intersection motive of certain Shimura varieties: the case of Siegel threefolds, 2017, 34 pages, available on arXiv.org under https://arxiv.org/abs/1706.02743

[W12] J. Wildeshaus, Poids maximaux de représentations, et poids du bord de variétés de Shimura de type PEL, in preparation. 